D. Popescu

Nagoya Math. J.

Vol. 100 (1985), 97-126

\title{
GENERAL NÉRON DESINGULARIZATION
}

\author{
DORIN POPESCU*)
}

\section{$\S 1$. Introduction}

Let $R \subset R^{\prime}$ be an "unramified" extension of discrete valuation rings in the sense that a local parameter $p$ of $R$ is also a local parameter in $R^{\prime}$. Suppose that the inclusion $R \rightarrow R^{\prime}$ induces separable extensions on fraction and residue fields. Then

(1.1) Theorem (Néron $[\mathrm{N}]) . \quad R^{\prime}$ is a filtered inductive limit of its finite type smooth sub-R-algebras.

In other words every finite type sub- $R$-algebra $B$ of $R^{\prime}$ can be embedded in a smooth finite type sub-R-algebra $B^{\prime}$ of $R^{\prime}$.

Let $T$ be a valuation ring containing a field $k$ of characteristic zero. Then

(1.2) Theorem (Zariski [Z]). T is a filtered inductive limit of the finite type smooth sub-k-algebras.

Actually the above result is stated only in the case when the fraction field of $T$ is an algebraic function field over $k$; but always we can reduce the problem to this case because the fraction field of $T$ is certainly a filtered inductive union of algebraic function fields over $k$.

Theorems as above are very useful when we want to reduce the solvability in $R^{\prime}$ (resp. T) of some polynomial equations over $R$ (resp. k) to the solvability of some polynomial equations for which it is possible to apply the Implicit Function Theorem. For this reason many results of Artin approximation theory are based on them. Attempts for extensions of these theorems were made in $[\mathrm{KMPPR}] \mathrm{Ch} . \mathrm{V},\left[\mathrm{P}_{1}\right],[\mathrm{CP}]$ and $\left[\mathrm{P}_{2}\right]$ but they preserved too much from Néron's case. In $\left[\mathrm{P}_{2}\right]$ (4.2.1) we put the following:

Received June 11, 1984.

*) Supported in part by INCREST and National Science Foundation Grant MCS18723 A03. 
(1.3) Question. Is a regular morphism a filtered inductive limit of finite type smooth morphisms?

Let $u: A \rightarrow A^{\prime}$ be a morphism of noetherian rings (all the rings considered here are supposed to be commutative with identity). Then $u$ is a filtered inductive limit of finite type smooth morphisms iff for every finite type $A$-algebra $B$ and every $A$-morphism $f: B \rightarrow A^{\prime}$ there exist a finite type smooth $A$-algebra $B^{\prime}$ and two $A$-morphisms $g: B \rightarrow B^{\prime}, h: B^{\prime} \rightarrow A^{\prime}$ such that $h g=f$ (apply e.g. [AD] Lemma (5.2)). Roughly speaking $\left(B^{\prime}, g, h\right)$ is a "desingularization" (in fact smoothification) of $(B, f)$ and we can reformulate the above Question in the following form:

(1.4) Question. Let $(B, f)$ be as above and suppose that $u$ is a regular morphism. Then has $(B, f)$ a "desingularization"?

This Question has already some positive answers in higher dimensions in the case when $u$ is the inclusion

i) $K\{X\} \rightarrow K \llbracket X \rrbracket, X=\left(X_{1}, \cdots, X_{n}\right)$, where $K$ is a nontrivial valued field of characteristic zero and $K\{X\}$ is the convergent power series ring in $X$ over $K$ (see [Pł], or [A])

ii) $K\langle X\rangle \rightarrow K \llbracket X \rrbracket$, where $K\langle X\rangle$ is the algebraic power series ring in $X=\left(X_{1}, \cdots, X_{n}\right)$ over an arbitrary field $K$ (see [A]).

In [A] M. Artin put the following

(1.5) Conjecture. Let $u ; A \rightarrow A^{\prime}$ be a regular morphism of excellent local rings, $B$ a finite type $A$-algebra, $D \subset$ Spec $B$ the (open) smooth locus of $B$ over $A$ and $f: B \rightarrow A^{\prime}$ an $A$-morphism. Then there exists a finite type smooth $A$-algebra $B^{\prime}$ and two $A$-morphisms $g: B \rightarrow B^{\prime}, h: B^{\prime} \rightarrow A^{\prime}$ such that $h g=f$ and $B^{\prime}$ is smooth over $B$ at $\tilde{h}\left(\tilde{f}^{-1}(D)\right)$, where $\tilde{h}$ : Spec $A$ $\rightarrow$ Spec $B^{\prime}, \tilde{f}:$ Spec $A^{\prime} \rightarrow \operatorname{Spec} B$ are given by $h, f$.

A recent progress is made by the following result (see [AD]):

(1.6) Theorem (M. Artin-J. Denef). The Conjecture (1.5) holds when either

1) $A=A^{\prime}, u=1_{A}$ and $A$ is a normal domain, or

2) $A^{\prime}$ is an excellent, normal, henselian local ring of dimension two and $u$ induces the trivial extension on residue fields.

Moreover V. Nica [Ni] proved that a regular morphism of noetherian (not necessarily normal) domains of dimension one is a filtered inductive 
limit of finite type smooth morphisms. Also he slightly improved Theorem (1.6).

Our aim is to give a positive answer to Question (1.4) (and so to (1.3)). Here we show this under some conditions of separability, for instance in the case when $A$ contains a field of characteristic zero (see Theorem (5.2) and Corollary (5.4)). The proof is mainly contained in Sections 7-9. The Desingularization Principle (Section 7) is very technical and so in order to get an idea of it we feel necessary to present it first on Néron's case (Section 6). Some easy examples of desingularization are given in Section 4 and in Sections 2-3 we give some preliminaries. This paper forms an improved extended version of a part of $\left[\mathrm{P}_{3}\right]$.

We would like to thank Professor M. Artin for his very helpful talks. Also we would like to thank The Institute for Advanced Study for their hospitality during the decissive stages of this work, their excellent conditions and stimulative atmosphere.

I express also my thanks to V. Nica, N. Radu and C. Rotthaus for useful conversations on the subject.

\section{$\S 2$. The smooth locus of an algebra}

(2.1) Let $f=\left(f_{1}, \cdots, f_{m}\right)$ be a system of polynomials in some variables $Y=\left(Y_{1}, \cdots, Y_{n}\right)$ over a ring $A$. For a system $g=\left(g_{1}, \cdots, g_{r}\right), r \leq n$ of $r$-polynomials from the ideal $(f)$, we consider the ideal $\Delta_{g}$ generated in $A[Y]$ by all $r \times r$-minors of the Jacobian matrix $(\partial g / \partial y)$ associated to $g$. Denote $H_{f}:=\sqrt{(f)+\sum_{g} \Delta_{g}((g):(f))}$, where the sum is taken over all systems $g$ of $r$-polynomials from $(f), r$ being variable positive integer and at most $n$. By Jacobian criterion of smoothness ([M] (29. E)), $V\left(H_{f}\right)$ is just the nonsmooth locus of $B:=A[Y] /(f)$, i.e. $B_{q}, q \in \operatorname{Spec} B$ is smooth over $A$ iff $q \not \supset H_{f} B$. Then for every finite presentation $A$-algebra $B$, let us say $B \cong A[Y] /(f)$, we can define an ideal $H_{B / A}:=H_{f} B \subset B$ which does not depend of the presentation chosen for $B$ over $A$.

(2.2) Note (Composition). Let $w: B \rightarrow C$ be a morphism of finite presentation $A$-algebras. Then it holds

$$
w\left(H_{B / A}\right) C \cap H_{C / B} \subset H_{C / A} \text {. }
$$

Indeed, let $q \in \operatorname{Spec} C, q \nsupseteq w\left(H_{B / A}\right) C \cap H_{C / B}$. Then $w^{-1} g \nsupseteq H_{B / A}$ and $q \nsupseteq H_{C / B}$ and so $B_{w-1 q}$ is smooth over $A$ and $C_{q}$ is smooth over $B$. Thus $C_{q}$ is smooth over $A$ i.e. $q \ngtr H_{C / A}$. 
(2.3) Note (Base change). Let $B, C$ be two $A$-algebras and $D:=$ $B \otimes{ }_{A} C$. Suppose that $B$ is of finite presentation over $A$. Then

$$
H_{B / A} D \subset H_{D / C} \text {. }
$$

Indeed, let $w: B \rightarrow D$ be the canonical map and $q \subset D$ a prime ideal which does not contain $H_{B / A} D$. Then $w^{-1} q \downarrow H_{B / A}$ and so $B_{w-1 q}$ is smooth over $A$. By base change $B_{w-1_{q}} \otimes_{A} C$ is smooth over $C$. Thus $D_{q}$ is smooth over $C$ and so $q \downarrow H_{D / C}$.

(2.4) Lemma. Let $u: A \rightarrow A^{\prime}$ be a morphism of rings, $B$ a finite presentation A-algebra, $x \in B$ an element and $f: B \rightarrow A^{\prime}$ an A-morphism. Suppose that

$$
f(x) \in \sqrt{f\left(H_{B / A}\right) A^{\prime}} .
$$

Then there exist a finite presentation A-algebra $C$ and two A-morphisms $v: B \rightarrow C, w: C \rightarrow A^{\prime}$ such that

i) $v(x) \in H_{C / A}$,

ii) $w v=f$,

iii) $v\left(H_{B / A}\right) C \subset H_{C / B}$ (in particular $H_{B / A} C \subset H_{C / A}$ (see Note (2.2))).

Proof. Let $b=\left(b_{1}, \cdots, b_{s}\right)$ be a system of generators of $H_{B / A}$. By hypothesis we have

$$
f(x)^{n}=\sum_{i=1}^{s} f\left(b_{i}\right) z_{i}
$$

for a certain positive integer $n$ and some elements $z=\left(z_{1}, \cdots, z_{s}\right)$ from $A^{\prime}$. Put $C:=B[Z] /\left(x^{n}-\sum_{i=1}^{s} b_{i} Z_{i}\right), Z=\left(Z_{1}, \cdots, Z_{s}\right)$ and let $w: C \rightarrow A^{\prime}$ be the extension of $f$ given by $Z \backsim z$. Clearly one has $b_{i} \in H_{C / B}$ and so the structure morphism $v$ of $C$ over $B$ satisfies iii). Since $v\left(x^{n}\right) \in \sum_{i=1}^{s} b_{i} C \subset H_{C / B}$ we get i).

Q.E.D.

(2.4.1) Note. Let $D:=\operatorname{Spec} B \backslash V\left(H_{B / A}\right)$. Then iii) says in fact that $C$ is smooth over $B$ at $\tilde{v}^{-1}(D)$ (compare with (1.5)), $\tilde{v}$ being given by $v$.

(2.5) Lemma. Let $B$ be a finite presentation A-algebra let us say $B \cong A[Y] /(f), \quad Y=\left(Y_{1}, \cdots, Y_{n}\right), f=\left(f_{1}, \cdots, f_{m}\right)$, the A-isomorphism being given by $Y \backsim y \in B^{n}$. Suppose that $A$ is regular and $B$ is a domain. Then

$$
H_{B / A}=\sqrt{\sum_{g} \Delta_{g}(Y)}
$$


where the sum is taken over all systems $g$ of $r$-polynomials, $r:=h t(f)$ from (f).

Proof. Indeed, let $q$ be a prime ideal from $B$ such that $q \searrow \sum_{g} \Delta_{g}(y)$ and $Q \subset A[Y], Q \supset(f)$ the prime ideal corresponding to $q$ by the above isomorphism. Then there exists a system $g=\left(g_{1}, \cdots, g_{r}\right)$ of polynomials from $(f)$ such that $\Delta_{g} \leftarrow Q$. By Jacobian criterion of regularity [M] (40.A), the ring $(A[Y] /(g))_{Q}$ is regular of dimension ht $Q-r$. In particular $g \cdot A[Y]_{Q}$ is a prime ideal of height $r$ and so $g \cdot A[Y]_{Q}=(f) A[Y]_{Q}$. Thus $((g):(f)) \not \subset Q$ and we get $\Delta_{g}((g):(f)) \not \subset Q$. i.e. $H_{B / A} \not \subset q$. Hence

$$
\sqrt{\sum_{g} \Delta_{g}(y)} \subset H_{B / A} \text {. }
$$

Conversely, let $h$ be a system of e-polynomials from $(f)$ such that $\left(\Delta_{h}((h):(f))\right)(y) \neq 0$. By $[\mathrm{M}](40 . \mathrm{A})$ the local ring $A[Y]_{(f)} /(h)$ is regular of dimension $r-e$ because $\Delta_{h}(y) \neq 0$. Since $((h):(f))(y) \neq 0$ it follows $r=e$ and we are ready because

$$
\left(\Delta_{h}((h):(f))\right)(y) \subset \Delta_{h}(y) .
$$

(2.6) Note. The above well known Lemma has in $\left[\mathrm{P}_{4}\right]$ the following extension (we do not use it here):

"Let $A \subset B$ be two reduced rings such that the inclusion $A \subset B$ is of finite presentation and preserves nonzero divisors; let us say $B \cong$ $A[Y] /(f), Y=\left(Y_{1}, \cdots, Y_{n}\right), f=\left(f_{1}, \cdots, f_{m}\right)$, the $A$-isomorphism being given by $Y \sim y \in B^{n}$. Suppose that

1) $\mathrm{A}$ is normal,

2) the minimal prime ideals of $B$ form a finite set $\operatorname{Min} B$, Then

3) $t:=\operatorname{trdeg}_{A_{q \cap A}} B_{q}=$ constant for all $q \in \operatorname{Min} B$.

$$
H_{B / A}=\sqrt{ } \sum_{g} \Delta_{g}(y),
$$

the sum being taken over all systems $g$ of $(n-t)$-polynomials from $(f)$ "

\section{§3. Standard elements for algebras}

(3.1) Let $B$ be a finite presentation algebra over a ring $A$; let us say $B \cong A[Y] / a, Y=\left(Y_{1}, \cdots, Y_{n}\right)$, the $A$-isomorphism being given by $Y \sim y=\left(y_{1}, \cdots, y_{n}\right) \in B^{n}$. In concrete situations when we want to apply some variants of the Newton Lemma we find difficult to deal with elements $d$ from $H_{B / A}$ unless there exists a system of polynomials $g=\left(g_{1}, \cdots, g_{r}\right)$ 
from a such that $d \in \sqrt{\left.\Delta_{g}((g): \mathfrak{a})\right)(y)}$; in this case we say that $d$ is a standard element for the presentation $B \cong A[Y] / \mathfrak{a}$. If $B$ has a presentation for which $d$ is a standard element then we say that $d$ is a standard element for $B$ over $A$. When 1 is a standard element for $B$ over $A$ than we call $B$ standard smooth over $A$. Thus standard smooth algebras are in particular smooth of finite presentation. Note that a multiple of a standard element is still a standard element.

Let $E$ be a Cohen algebra over a noetherian local ring $(D, \mathfrak{m})$, i.e.

1) $E$ is flat over $D$,

2) $(E, \mathfrak{m} E)$ is a noetherian complete local ring,

3) the residue field extension $D / \mathfrak{m} \rightarrow E / \mathfrak{m} E$ is separable.

(3.2) Lemma. $E$ is the completion of a noetherian local ring $F$ which is a filtered inductive limit of some standard smooth sub-D-algebras of $E$.

Proof. Using a transfinite induction argument it is easy to show that $E$ is a completion of a filtered inductive limit $F$ of some sub- $D$-algebras of $E$ of the form $D[Y]_{m}$ or $G_{\mathrm{m} G}, G:=D[Y]_{\mathrm{m}}[Z] /(f), Y=\left(Y_{1}, \cdots, Y_{n}\right), n \in N$, where $f$ is a monic polynomial in $Z$ inducing modulo $m$ an irreducible separable one. Thus it is enough to see that $D[Y]_{\mathrm{m}}$ and $G_{\mathrm{m} G}$ are filtered inductive limits of some standard smooth sub- $D$-algebras of $E$ of the form $D[Y]_{g}$, resp. $\left(D[Y]_{g}[Z] /(f)\right)_{h}$, where $g \in D[Y] \backslash m D[Y], h \in D[Y]_{g}[Z]$ being a multiple of $\partial f / \partial z$.

Q.E.D.

Since artinian local rings are complete, Question (1.3) has a positive answer in artinian rings.

(3.3) Conollary. Let $A \rightarrow A^{\prime}$ be a regular local morphism of artinian local rings. Then $A^{\prime}$ is a filtered inductive limit of its standard smooth sub-A-algebras,

The following result is inspired from [E].

(3.4) Lemma. Let $A$ be a noetherian ring, $A^{\prime}, B$ two $A$-algebras the last one being of finite type and $u: B \rightarrow A^{\prime}$ an $A$-morphism. Then there exist a finite type A-algebra $C$ and two A-morphisms $v: B \rightarrow C, w: C \rightarrow A^{\prime}$ such that

i) $w v=u$,

ii) $v\left(H_{B / A}\right) \subset H_{C / B}$ (in particular $\left.v\left(H_{B / A}\right) \subset H_{C / A}\right)$,

iii) there exists a presentation of $C$ over $A$ for which all elements of $v\left(H_{B / A}\right)$ are standard. 
Proof. Suppose that $B \cong A[Y] /(f), \quad Y=\left(Y_{1}, \cdots, Y_{n}\right), f=\left(f_{1}, \cdots, f_{m}\right)$, the $A$-isomorphism being given by $Y \backsim y \in B^{n}$ and choose a system of generators $p_{k}=\left(p_{k 1}, \cdots, p_{k m}\right), k=1, \cdots, e$ for the kernel of the map $A[Y]^{m} \rightarrow(f) /(f)^{2}$ given by $\left(L_{1}, \cdots, L_{m}\right) \backsim \sum_{l=1}^{m} L_{i} \hat{f}_{i}$, where " $\wedge$ " denotes the residue modulo $(f)^{2}$. Put $h_{h}:=\sum_{i=1}^{m} p_{k i} Z_{i}, k=1, \cdots, e, C:=B[Z] /(h)$, $Z=\left(Z_{1}, \cdots, Z_{m}\right), h=\left(h_{1}, \cdots, h_{e}\right)$ and let $w: C \rightarrow A^{\prime}$ be the map extending $u$ by $Z \backsim 0$ and $v$ the composite map $B \rightarrow B[Z] \rightarrow C$.

Fix an element $x \in H_{B / A}$. Then $B_{x}$ is a smooth $A$-algebra and so $E:=(f) /(f)^{2} \otimes_{B} B_{x}$ is a projective $B_{x}$-module. Since $C_{v(x)}$ is the symmetric $B_{x}$-algebra associated to $E$ we deduce that $C_{v(x)}$ is a smooth $B_{x}$-algebra and so $v(x) \in H_{C / B}$, i.e. ii) holds.

Let $Y^{\prime}=\left(Y_{1}^{\prime}, \cdots, Y_{n}^{\prime}\right)$ be some new variables and $I=\left(f, Y^{\prime}, h\right) A\left[Y, Y^{\prime}, Z\right]$. We claim that the elements of $H_{B / A}$ are standard for the presentation $C \cong A\left[Y, Y^{\prime}, Z\right] / I$ of $C$ over $A$. Indeed, let $d \in H_{B / A}$ and $F \in A[Y]$ a polynomial such that $d=F(y)$. The above construction of $I$ from $(f)$ is exactly the one considered in the proof of Elkik's Theorem (see [E] or [KMPPR] $(I ; 6.1))$ and like there we get that $I_{F^{\prime}} / I_{F^{\prime}}^{2}$ is a free $C_{d} \cong\left(A\left[Y, Y^{\prime}, Z\right] / I\right)_{F^{-}}$ module. Let $g=\left(g_{1}, \cdots, g_{r}\right)$ be a system of polynomials from $I$ inducing a base in $I_{F} / I_{F}^{2}$. Thus we have

$$
I_{F}=(g) A\left[Y, Y^{\prime}, Z\right]_{F}+I_{F}^{2} .
$$

By Nakayama's Lemma there exists an element $\alpha$ from $1+I_{F^{\prime}}$ such that $\alpha I_{F} \subset(g) A\left[Y, Y^{\prime}, Z\right]_{F}$, i.e. there exist a certain positive integer $t$ and a polynomial $M \in F^{t}+I$ such that $M I \subset(g)$. Thus $v(d) \in \sqrt{((g): I)(y, 0, z)}$, where $z$ is the element induced by $Z$ in $C$. Since $C_{v(d)}$ is smooth over $A$ we get also $F \in \sqrt{\Delta_{g}}$ and so

$$
v(d) \in \sqrt{\left(\Delta_{g}((g): I)\right)(y, 0, z)}
$$

i.e. iii).

Q.E.D.

(3.4.1) Note. In general the presentation $C \cong A[Y, Z] /(f, h)$ does not satisfy iii) from the above lemma. Thus an element can be standard for a presentation and nonstandard for another one.

(3.5) Corollary. Let $A$ be a noetherian ring, $A^{\prime}, B$ two A-algebras, the last one being smooth of finite type and $u: B \rightarrow A^{\prime}$ an A-morphism. Then there exist a standard smooth A-algebra $C$ and two A-morphisms $v: B \rightarrow C, w: C \rightarrow A^{\prime}$ such that 
i) $w v=u$

ii) $C$ is smooth over $B$.

(3.6) Corollary. Let $A$ be a noetherian ring, $A^{\prime}, B$ two $A$-algebras and $u: B \rightarrow A^{\prime}$ an $A$-morphism. Suppose that $B$ is of finite type over $A$ and

$$
u\left(H_{B / A}\right) A^{\prime}=A^{\prime} .
$$

Then there exist a standard smooth A-algebra $C$ and two A-morphisms $v: B \rightarrow C, w: C \rightarrow A^{\prime}$ such that

i) $w v=u$,

ii) $v\left(H_{B / A}\right) \subset H_{C / B}$.

For the proof apply Lemma (2.4) for $x=1 \in B$ and then the result follows from Corollary (3.5).

\section{§4. Examples of desingularization: the linear case}

(4.1) ExAmple. Let $(A, \mathfrak{m})$ be a two dimensional regular local ring and $\{a, b\}$ a regular system of parameters in $A$. Let $A^{\prime}$ be a domain-flat $A$-algebra and $x, y \in A^{\prime}$ two elements such that

1) $\operatorname{trdeg}_{A} B=1, B:=A[x, y]$,

2) $a x+b y=0$.

By flatness, $\{a, b\}$ is still a regular sequence in $A^{\prime}$ and so every solution of the linear equation

3) $a X+b Y=0$

in $A^{\prime}$ is a multiple of $(-b, a)$. In particular there exists $z \in A^{\prime}$ such that

4) $x=-b z, y=a z$.

Put $f:=a X+b Y, B^{\prime}:=A[z]$. By 4) we have $B^{\prime} \supset B$ and $\operatorname{trdeg}_{A} B^{\prime}=1$ (see 1)). Thus $B^{\prime}$ is a polynomial $A$-algebra in $z$ (in particular standard smooth) containing $B$. Note that

5) $H_{B / A} \supset \Delta_{f}(x, y)=(a, b) B=\mathfrak{m} B$

by Lemma (2.5).

More general examples are given by the following Lemma which is not far from some facts contained in [AD] p. 8-9.

(4.2) Lemma. Let $A$ be a noetherian domain, $E=\left(e_{i j}\right)_{\substack{1 \leq i \leq m \\ 1 \leq j \leq n}}$ a matrix of rank $r$ over $A, f=\left(f_{1}, \cdots, f_{m}\right), f_{i}:=\sum_{j=1}^{n} e_{i j} Y_{j}, Y=\left(Y_{1}, \cdots, Y_{n}\right), B:=$ $A[Y] /(f), A^{\prime}$ a flat A-álgebra, $u: B \rightarrow A^{\prime}$ an A-morphisn and $I \subset A$ the ideal generated by all $r \times r$-minors of $E$. Then

i) $H_{B / A} \supset I B$, 
ii) there exist a positive integer $t$ and two A-morphisms $v: B \rightarrow B^{\prime}:=$ $A[X], X=\left(X_{1}, \cdots, X_{t}\right), w: B^{\prime} \rightarrow A^{\prime}$ such that

$\left.\mathrm{ii}_{1}\right) \quad w v=u$,

$\left.\mathrm{ii}_{2}\right) H_{B^{\prime} / B} \supset I B^{\prime}$.

Proof. Let $d$ be a nonzero $r \times r$-minor of $E$. Suppose $d$ is given on first $r$-rows. Then $d f_{j} \in\left(f_{1}, \cdots, f_{r}\right)$ for all $j>r$ and so $d^{2} \in \Delta_{f_{1}, \cdots, f_{r}}\left(\left(f_{1}, \cdots, f_{r}\right):(f)\right)$, i.e. i) holds.

Let $a_{k}=\left(a_{k j}\right)_{1 \leq j \leq n}, k=1, \cdots, t$ be a complete system of solutions of $f$ in $A$. By flatness $\left\{a_{k}\right\}_{k}$ induces a complete system of solutions of $f$ in $A^{\prime}$ and so there exist some elements $x=\left(x_{1}, \cdots, x_{t}\right)$ from $A^{\prime}$ such that

$$
u\left(y_{j}\right)=\sum_{k=1}^{t} a_{h \jmath} x_{k},
$$

where $y_{j} \in B$ is induced by $Y_{j}$. The system of polynomials $h=\left(h_{1}, \cdots, h_{n}\right)$, $h_{j}:=\sum_{k=1}^{t} a_{k j} X_{k}$ form in $B^{\prime}:=A[X], X=\left(X_{1}, \cdots, X_{t}\right)$ a solution of $f$ and so we get an $A$-morphism $v: B \rightarrow B^{\prime}$ by $Y \sim h$. Thus the $A$-morphism $w: B^{\prime} \rightarrow A^{\prime}$ given by $X \backsim x$ satisfies $\mathrm{ii}_{1}$ ).

Denote $g=\left(g_{1}, \cdots, g_{n}\right), g_{l}:=y_{l}-h_{j} \in B[X]$. We have the following $A$-isomorphisms:

$$
B[X] /(g) \cong A[X, Y] /(f, Y-h) \cong A[X]
$$

Let $s$ be the rank of the $t \times n$-matrix $\left(a_{k j}\right)$ and $J \subset A$ the ideal generated by all $s \times s$-minors of them. By i) we have

$$
H_{B^{\prime} / B} \supset J B^{\prime}
$$

and so it is enough to show the following elementary Lemma:

(4.2.1) LemMa $I \subset \sqrt{ } \bar{J}$.

Proof. Let $\alpha: A^{t} \rightarrow A^{n}, \beta: A^{n} \rightarrow A^{m}$ be the linear maps given by $\left(a_{h j}\right)$ resp. E. Clearly the sequence $A^{t} \stackrel{\alpha}{\longrightarrow} A^{n} \stackrel{\beta}{\longrightarrow} A^{m}$ is exact, $\left\{a_{k}\right\}_{k}$ being a system of generators for $\operatorname{Ker} \beta$ and so we have $s+r=n$.

Let $M$ be a $r \times r$-minor of $E$. We claim that $1 \in J A_{M}$. Indeed otherwise there exists a maximal ideal $q \subset A_{M}$ containing $J$. By Cramer's rule note that the image of $A_{q} \otimes_{A} \beta$ is a free module of rank $r$. Then the following sequence

$$
k(q)^{t} \stackrel{k(q(\otimes \alpha}{\longrightarrow} k(q)^{n} \longrightarrow k(q)^{r} \longrightarrow 0
$$


is exact, $k(q):=A_{q} / q A_{q}$. It follows

$$
r k(k(q) \otimes \alpha)=n-r=s
$$

which contradicts the choice of $q$ !. Then $M \in \sqrt{ } \bar{J}$

Q.E.D.

(4.3) Corollary. Let $A, E, Y, f, I$ be like in Proposition (4.2), $b=$ $\left(b_{1}, \cdots, b_{m}\right)$ a system of elements from $A, B:=A[Y] /(f-b), A^{\prime}$ a faithfully flat A-algebra and $u: B \rightarrow A^{\prime}$ an A-morphism. Then

i) $H_{B / A} \supset I B$

ii) there exist a positive integer $t$ and two A-morphisms $v: B \rightarrow B^{\prime}:=$ $A[X], X=\left(X_{1}, \cdots, X_{t}\right), w: B^{\prime} \rightarrow A^{\prime}$ such that

$\left.\mathrm{ii}_{1}\right) \quad w v=u$,

$\left.\mathrm{ii}_{2}\right) \quad H_{B^{\prime} / B} \supset I B^{\prime}$.

Proof. By faithfully flatness the system of linear equations

$$
f(Y)=b
$$

has a solution $\tilde{y}$ in $A$ because it has one in $A^{\prime}$. Then there exists an $A$-isomorphism $B \cong A[Y] /(f)$ given by $Y \backsim Y+\tilde{y}$. Now it is enough to apply Proposition (4.2).

Q.E.D.

\section{§5. Main results}

An important part of our paper Sections $7-9$ is devoted to the proof of the following:

(5.1) Desingularization Lemma. Let $u: A \rightarrow A^{\prime}$ be a morphism of noetherian rings, $B$ a finite type A-algebra, $f: B \rightarrow A^{\prime}$ a morphism of $A$ algebras and $q$ a minimal prime over-ideal of $\mathfrak{a}:=\sqrt{f\left(\bar{H}_{B / A}\right) A^{\prime}}$. Suppose that:

i) the field extension $k\left(u^{-1} q\right) \subset k(q)$ is separable (as usual $k(q)$ denotes the fraction field of $\left.A^{\prime} / q\right)$,

ii) for every minimal prime over-ideal $p$ of a the map $A \rightarrow A_{p}^{\prime}$ induced by $u$ is flat and the ring $\left.A_{p}^{\prime} / u^{-1} p\right) A_{p}^{\prime}$ is regular,

iii) $A^{\prime} / q$ is not finite.

Then there exist a finite type A-algebra $B^{\prime}$ and two A-morphisms $v: B \rightarrow B^{\prime}$, $w: B^{\prime} \rightarrow A^{\prime}$ such that
(*) $\quad w v=f$
$(* *) \quad a \subset \sqrt{w\left(H_{B^{\prime} / A}\right) A^{\prime}} \not \subset q$ 
(5.2) Theorem. Let $u: A \rightarrow A^{\prime}$ be a morphism of noetherian rings and $F \subset$ Spec $A^{\prime}$ a closed set such that for every prime ideal $q \in F$

i) the field extension $k\left(u^{-1} q\right) \subset k(q)$ is separable

ii) the map $A \rightarrow A_{q}^{\prime}$ induced by $u$ is formally smooth.

Then for every finite type A-algebra $B$ and every morphism of A-algebras $f: B \rightarrow A^{\prime}$ such that

$V\left(\mathfrak{a}_{f}\right) \subset F, \mathfrak{a}_{f}:=f\left(H_{B / A}\right) A^{\prime}$ there exist a standard smooth A-algebra $B^{\prime}$ and two A-morphisms $v: B \rightarrow B^{\prime}, w: B^{\prime} \rightarrow A^{\prime}$ such that $w v=f$.

Proof. If $F=\emptyset$ then the result is a consequence of Corollary (3.6). Apply noetherian induction on $F$. Let $(B, f)$ be such that $V\left(\mathfrak{a}_{f}\right) \subset F$. Choose a minimal prime ideal associated to $\mathfrak{a}_{f}$. First suppose infinite the residue fields of all prime ideals from $F$. Applying Desingularization Lemma for $(B, f, q)$ we find a finite type $A$-algebra $B_{1}$ and two $A$-morphisms $h: B \rightarrow B_{1}, f_{1}: B_{1} \rightarrow A^{\prime}$ such that

1) $f_{1} h=f$

2) $\mathfrak{a}_{f_{1}} \not \subset q$ and so $V\left(\mathfrak{a}_{f_{1}}\right) \subsetneq V\left(a_{f}\right)$

By the induction hypothesis there exist a standard smooth $A$-algebra $B^{\prime}$ and two $A$-morphisms $v_{1}: B_{1} \rightarrow B^{\prime}, w: B^{\prime} \rightarrow A^{\prime}$ such that $w v_{1}=f_{1}$. Take $v:=v_{1} h$ and we are ready.

If $F$ contains prime ideals whose residue fields are finite then consider the composite map $u^{\prime}: A \stackrel{u}{\longrightarrow} A^{\prime} \stackrel{j}{\longrightarrow} A^{\prime}[X]_{X}$. Like above we find a standard smooth $A$-algebra $B^{\prime}$ and two $A$-morphisms $v: B \rightarrow B^{\prime}, w^{\prime}: B^{\prime} \rightarrow A^{\prime}[X]_{X}$ such that $w^{\prime} v=j f$. Then let $w$ be the composite map $B^{\prime} \stackrel{w^{\prime}}{\longrightarrow} A^{\prime}[X]_{X} \rightarrow A^{\prime}$, the last map being given by $X \rightarrow 1$.

Q.E.D.

(5.2.1) We say that the couple $(B, f)$ has a desingularization (with respect to $u$ ) if there exists a standard smooth $A$-algebra $B^{\prime}$ and two $A$ morphisms $v: B \rightarrow B^{\prime}, w: B^{\prime} \rightarrow A^{\prime}$ such that $w v=f$. This is a particular case of the "desingularization" introduced in Section 1.

(5.3) Corollary. Let $A$ be a noetherian ring, $a \subset A$ an ideal, $\hat{A}$ the completion of $A$ in the a-adic topology. $B$ a finite type A-algebra and $f: B$ $\rightarrow \hat{A}$ a morphism of A-algebras. Suppose that $V(a \hat{A})=V\left(f\left(H_{B / A}\right) \hat{A}\right)$. Then $(B, f)$ has a desingularization.

Proof. Since $A / \mathfrak{a} \cong \hat{A} / \mathfrak{a} \hat{A}$ the extension $k(q \cap A) \subset k(q)$ is trivial for every $q \in V(a \hat{A})$. Clearly $A \rightarrow \hat{A}$ is subject to i) and ii) from Theorem (5.2) which we can apply now.

Q.E.D. 
(5.4) Corollary. Let $A \rightarrow A^{\prime}$ be a regular morphism of noetherian rings, $B$ a finite type A-algebra and $f: B \rightarrow A^{\prime}$ a morphism of $A$-algebras. Suppose that $A$ contains a field of characteristic zero. Then $(B, f)$ has a desingularization.

\section{$\S 6$. An idea of the proof of Desingularization Lemma in Néron's case}

The aim of this section is not to give a new proof to Néron's $p$ desingularization. Actually we prove here considerably less in a more difficult way. But the ideas of our Desingularization Lemma came from Néron's case and it is easier to understand them, "historically" speaking, on this case.

Let $A \subset A^{\prime}$ be an unramified extension of discrete valuation rings, $v: A^{\prime} \backslash\{0\} \rightarrow Z$ the valuation of $A^{\prime}$ and $p \in A$ a local parameter in $A$ (and $\left.A^{\prime}\right)$. Suppose that $\operatorname{trdeg}_{A} A^{\prime}=\infty$ and the inclusion $A \longrightarrow A^{\prime}$ induces a separable extension on fraction and residue fields. Let $B:=A[y], y=$ $\left(y_{1}, \cdots, y_{N}\right), y \in A^{\prime N}$ be a finite type sub- $A$-algebra of $A^{\prime}$. Since $Q(B) \supset Q(A)$ is a separable field extension $(Q(R)$ denotes in our paper the fraction field of a domain $R$ ), we get $H_{B / A} \neq(0)$. If $H_{B / A} A^{\prime}=A^{\prime}$ then $H_{B / A} \not \subset p A^{\prime}$ and by Jacobian criterion of smoothness [M] (29.E) there exists a system $f$ of $r$ polynomials, $r:=N$-trdeg ${ }_{A} B$ such that $f(y)=0$ and $\Delta_{f}(y) \not \subset p A^{\prime}$. Take an element $b \in \Delta_{f}(y) \backslash p A^{\prime}$. Then $B^{\prime}:=B_{b}$ is a standard smooth embedding of $B$ in $A^{\prime}$ (in general in this place we shall apply Corollary (3.6)). If $H_{B / A} A^{\prime} \neq A^{\prime}$ we shall reduce our problem to the previous case using the following

(6.1) Lemma. If $\sqrt{H_{B / A} A^{\prime}}=p A^{\prime}$ then there exists a finite type subA-algebra $B^{\prime}$ of $A^{\prime}$ containing $B$ such that $H_{B^{\prime} / A} A^{\prime}=A^{\prime}$.

Proof. Let $f$ be a system of $r$-polynomials in $Y=\left(Y_{1}, \cdots, Y_{N}\right)$ such that $f(y)=0$ and $\Delta_{f}(y) \neq 0$. Put $t=v\left(\Delta_{f}(y)\right)=\inf \left\{v(d) \mid d \in \Delta_{f}(y)\right\}>0$. Clearly there exists a $r \times r$-minor $M$ of the Jacobian matrix $J:=(\partial f / \partial r)$ such that $v(M(y))=t$.

Step 1. Case $B / p^{2 t+1} A^{\prime} \cap B \cong A / p^{2 t+1} A$

Thus there exist $y^{\prime} \in A^{N}, y^{\prime \prime} \in p A^{\prime N}$ such that

$$
y=y^{\prime}+p^{2 t} y^{\prime \prime}
$$


By Taylor's formula we get $M\left(y^{\prime}\right) \equiv M(y) \bmod p^{2 t} A^{\prime}$ and so $v\left(M\left(y^{\prime}\right)\right)=t$, i.e. $M\left(y^{\prime}\right)=p^{t} u$ for an unit element $u \in A$. Suppose that the minor $M$ is given on first $r$-columns. We complete $J$ to a square matrix $H$ by adding the last $(N-r)$-rows of the unit $N \times N$-matrix $I_{N}$ (if $r=N$ put $H:=J$ ). Then $\operatorname{det} H=M$ and so there exists a $N \times N$-matrix $G^{\prime}$ over $A[Y]$ such that $H G^{\prime}=G^{\prime} H=M I_{N}$. Let $G$ be the matrix obtained from $G^{\prime}$ multiplying its rows by $u^{-1}$. We have $H G=G H=\left(u^{-1} M\right) I_{N}$ and so

$$
H\left(y^{\prime}\right) G\left(y^{\prime}\right)=G\left(y^{\prime}\right) H\left(y^{\prime}\right)=p^{t} I_{N} .
$$

Take $z:=H(y) y^{\prime \prime}$. We have $p^{t} y^{\prime \prime}=G\left(y^{\prime}\right) \cdot z$ and thus

$$
y=y^{\prime}+p^{t} G\left(y^{\prime}\right) z .
$$

By Taylor's formula we obtain

$$
0=f(y)=f\left(y^{\prime}\right)+p^{t} J\left(y^{\prime}\right) G\left(y^{\prime}\right) z+p^{2 t} Q(z),
$$

where $Q=\left(Q_{i}\right)_{i} \in A[Z]^{r}, Z=\left(Z_{1}, \cdots, Z_{N}\right)$ is a system of $r$-polynomials containing just monomials of degree $\geq 2$. Note taht $J G=p^{t} E$, where $E=\left(I_{r} \mid O\right)$ : this follows from (6.1.2). Thus we get

$$
f_{i}\left(y^{\prime}\right)+p^{2 t}\left(z_{i}+Q_{i}(z)\right)=0, \quad i=1, \cdots, r
$$

and so $f_{i}\left(y^{\prime}\right) \in p^{2 t} A^{\prime} \cap A=p^{2 t} A$, i.e. there exist $c_{i} \in A$ such that $f_{i}\left(y^{\prime}\right)=p^{2 t} c_{i}$, $i=1, \cdots, r$. Then $z$ is a solution of the following system of polynomials over $A$ :

$$
g_{i}:=c_{i}+Z_{i}+Q_{i}(Z), \quad i=1, \cdots, r .
$$

Take $B^{\prime}:=A[z]$. From (6.1.3) it follows

$$
B \subset B^{\prime} \subset B_{p}
$$

and so $Q(B)=Q\left(B^{\prime}\right)$. Thus $r=N-\operatorname{trdeg}_{A} B^{\prime}$ and by Lemma (2.5) we get $\Delta_{g}(z) \subset H_{B^{\prime} / A}$ for $g=\left(g_{1}, \cdots, g_{r}\right)$. Let $P$ be the $r \times r$-minor of $(\partial g / \partial Z)$ given on first $r$-columns. Then $P(z) \in 1+z A^{\prime} \subset 1+y^{\prime \prime} A^{\prime} \subset 1+p A^{\prime}$ because $Q$ contains only monomials of degree $\geq 2$. As $P(z) \in H_{B^{\prime} / A}$ we get $H_{B^{\prime} / A} A^{\prime}=A^{\prime}$.

Step 2. Case when there exist two finite type sub-A-algebras $D \subset C \subset A^{\prime}$ such that

1) $B \subset D+p^{2 t+1} A^{\prime}$,

2) $p^{2 t} A^{\prime} \cap D \subset p^{2 t} C$,

3) $C$ is smooth over $A$,

4) the fields $Q(B)$ and $Q(C)$ are algebraically disjoint over $Q(A)$. 
This step is an extension of Step 1. Here we find the kernel of so called Desingularization Principle (Section 7). By 1) we can find $y^{\prime} \in D^{v}$, $y^{\prime \prime} \in p A^{\prime N}$ such that

$$
y=y^{\prime}+p^{2 t} y^{\prime \prime} .
$$

By Taylor's formula we get $M\left(y^{\prime}\right) \equiv M(y) \bmod p^{2 t} A^{\prime}$ and so $M\left(y^{\prime}\right)=p^{t} u$ for an unit $u \in A^{\prime}$. Fix an $i, 1 \leq i \leq 2 t$ and an element $\omega \in p^{i} A^{\prime} \cap D$. Then $p^{2 t-i} \omega \in p^{2 t} A^{\prime} \cap D \subset p^{2 t} C$ and so there exists an element $\alpha \in C$ such that $p^{2 t-i} \omega=p^{2 t} \alpha$ Since $A^{\prime}$ is a domain one has $\omega=p^{i} \alpha \in p^{i} C$. Thus

$$
p^{i} A^{\prime} \cap D \subset p^{i} C, \quad i=1, \cdots, 2 t .
$$

In particular we get $u \in C$. Like in Step 1 construct $H, G^{\prime}, G, z$. Now $G$ is a matrix over $C_{u}[Y]$ because $u \in C$. Changing $C$ by $C_{u}$ we can suppose that $G$ is a matrix over $C[Y]$. We have

$$
y=y^{\prime}+p^{t} G\left(y^{\prime}\right) z .
$$

By Taylor's formula we obtain

$$
0=f(y)=f\left(y^{\prime}\right)+p^{t} J\left(y^{\prime}\right) G\left(y^{\prime}\right) z+p^{2 t} Q(z),
$$

where $Q=\left(Q_{i}\right)_{i} \in C[Z]^{r}, Z=\left(Z_{1}, \cdots, Z_{N}\right)$ is a system of $r$-polynomials containing just monomials of degree $\geq 2$. Like in Step 1 we get

$$
f_{i}\left(y^{\prime}\right)+p^{2 t}\left(z_{i}+Q_{i}(z)\right)=0, \quad \text { if } i=1, \cdots, r
$$

and so $f_{i}\left(y^{\prime}\right) \in p^{2 t} A^{\prime} \cap D \subset p^{2 t} C$, i.e. there exist $c_{i} \in C$ such that $f_{i}\left(y^{\prime}\right)=p^{2 t} c_{i}$, $i=1, \cdots, r$. Then $z$ is a solution of the following system of polynomials over $C$ :

$$
g_{i}:=c_{i}+Z_{i}+Q_{i}(Z), \quad i=1, \cdots, r .
$$

Take $B^{\prime}:=C[z]$. We have $B^{\prime} \supset B$ and $Q\left(B^{\prime}\right)=Q(C[B])=Q(C)(Q(B))$. By 4) we get

$$
\operatorname{trdeg}_{Q(C)} Q\left(B^{\prime}\right)=\operatorname{trdeg}_{Q(A)} Q(B)=N-r
$$

and so $\Delta_{g}(z) \subset H_{B^{\prime} / C}$ by Lemma (2.5), $C$ being a regular ring because of 3). But $\Delta_{g}(z) \not \subset p A^{\prime}$ and so $H_{B^{\prime} / C} A^{\prime}=A^{\prime}$. As $C$ is smooth over $A$ we are ready by Note (2.2).

\section{Step 3. Reduction to Step 2}

Here we construct $D, C$ from Step 2. This procedure suggested to 
us the so called Lifting Lemma (see Section 8). Using Corollary (3.3) we can embed $\tilde{B}:=B / p^{2 t+1} A^{\prime} \cap B$ in a standard smooth sub- $\tilde{A}:=\left.A\right|^{2 t+1} A$ algebra $\tilde{D}$ of $\tilde{A}^{\prime}:=\tilde{A} \otimes_{A} A^{\prime}$. Then there exist a system of generators $\tilde{u}=\left(\tilde{u}_{i}\right)_{i=1, \ldots, n}$ from $\tilde{D}$ and a system of polynomials $\tilde{h}=\left(\tilde{h}_{1}, \cdots, \tilde{h}_{e}\right)$ from the kernel $\tilde{\mathfrak{a}}$ of the map $\tilde{A}[U] \rightarrow \tilde{D}, U=\left(U_{1}, \ldots, U_{n}\right) \backsim \tilde{u}$ such that $\left(\Delta_{\tilde{h}}((\tilde{h}): \tilde{a})\right)(\tilde{u})=\tilde{D}$. Let $u, h$ be some liftings of $\tilde{u}$ resp. $\tilde{h}$ to $A^{\prime}$ resp. $A[U]$. Adding to $u$, if it is necessary, some elements from $A^{\prime}$ of the form $p^{2 t+1} \beta, \beta=\left(\beta_{1}, \cdots, \beta_{n}\right)$ with $\operatorname{trdeg}_{B} B[\beta]=n\left(\operatorname{trdeg}_{A} A^{\prime}=\infty\right)$ we can suppose that $\operatorname{trdeg}_{B} B[u]=n$. Put $D=A[u]$. Since $\tilde{B} \subset \tilde{D}$ we have $B \subset D+p^{2 t+1} A^{\prime}$, i.e. 1). As $h(u) \in p^{2 t+1} A^{\prime}$, there exist some elements $w=\left(w_{1}, \cdots, w_{e}\right)$ such that $h(u)=p^{2 t+1} w$. Take $C^{\prime}:=D[w], h^{\prime}:=h-p^{2 t+1} W, W=\left(W_{1}, \cdots, W_{e}\right)$. Since $h^{\prime}(u, w)=0$ and $\operatorname{trdeg}_{Q(A)} Q\left(C^{\prime}\right)=n$ we get $\Delta_{h^{\prime}}(u, w) \subset H_{C^{\prime} / A}$ by Lemma (2.5). Thus $p \in H_{C^{\prime} / A}, H_{C^{\prime} / A} \cdot \tilde{A}^{\prime}=\tilde{A}^{\prime}$ and it follows

$$
H_{C^{\prime} / A} A^{\prime}=A^{\prime} \text {. }
$$

Let $x \in p^{2 t+1} A^{\prime} \cap D$. Then there exists a polynomial $P \in A[U]$ such that $x=P(u)$. Since $P(u) \equiv 0 \bmod p^{2 t+1} A^{\prime}$ the residue $\tilde{P}$ modulo $p^{2 t+1}$ of $P$ belongs to $\tilde{a}$. Then $((\tilde{h}): \tilde{P})(\tilde{u})=\tilde{D}$ and so there exists a polynomial $F \in A[U]$ such that $\mu:=F(u) \notin p A^{\prime}$ and $F P \in\left(p^{2 t+1}, h\right) A[U]$. In particular one has

$$
\mu x=(F P)(u) \in\left(p^{2 t+1}, h(u)\right) D \subset p^{2 t+1} C^{\prime} .
$$

Choose one element $\lambda \in H_{C^{\prime} / A}$ which is not $p A^{\prime}$ and put $C=C_{(\lambda \mu)}^{\prime}$. From (6.1.11) and (6.1.12) we get 3) and 2) (see (6.1.7)). Finally note that $Q(B) \otimes_{Q(A)} Q(C)$ is in fact a fraction ring of $B[U]$ and so 4$)$ holds too.

Q.E.D.

\section{§7. Desingularization Principle}

(7.1) Proposition. Let $A \rightarrow A^{\prime}$ be a ring morphism, $B$ a finite presentation A-algebra, $y=\left(y_{1}, \cdots, y_{N}\right)$ a system of generators of $B$ over $A$, $p$ the kernel of the map $A[Y] \rightarrow B, Y=\left(Y_{1}, \cdots, Y_{.}\right) \sim y, \alpha: B \rightarrow A^{\prime}$ an $A$ morphism, $d$ an element from $A, e \geq 2$ a positive integer and $\bar{A}:=A / d^{2 e+1} A$, $\bar{B}:=\bar{A} \otimes_{A} B, \bar{A}^{\prime}:=\bar{A} \otimes_{A} A^{\prime} . \quad$ Suppose that

i) $\mathrm{Ann}_{A} d^{e}=\mathrm{Ann}_{A} d^{e+1}, \mathrm{Ann}_{A^{\prime}} d^{e} A^{\prime}=\mathrm{Ann}_{A^{\prime}} d^{e+1} A^{\prime}$

ii) there exists a system of polynomials $f=\left(f_{1}, \cdots, f_{r}\right)$ from $p$ such that $d \in\left(\Delta_{f}((f): \mathfrak{p})\right)(y)$

iii) there exist a finite type A-algebra $D$, an ideal $\mathfrak{b} \subset D, \bar{D}:=D / \mathfrak{b}$ and 
two A-morphisms $\beta: D \rightarrow A^{\prime}, \bar{\gamma}: \bar{B} \rightarrow \bar{D}$ such that $d^{2 e+1} \in \mathfrak{b}$ and $\bar{\beta} \bar{\gamma}=\bar{\alpha}$, where $\bar{\alpha}: \bar{B} \rightarrow \bar{A}^{\prime}, \bar{\beta}: \bar{D} \rightarrow \bar{A}^{\prime}$ are given canonically by $\alpha$, respectively $\beta$,

iv) there exist a finite presentation A-algebra $C$ containing $D$ and an A-morphism $\rho: C \rightarrow A^{\prime}$ such that

$\left.\operatorname{iv}_{1}\right)\left.\quad \rho\right|_{D}=\beta$,

$\left.\mathrm{iv}_{2}\right) \quad \mathfrak{b} \subset d^{2 e+1} C$.

Then there exist a finite presentation $C$-algbera $B^{\prime}$ and two $A$-morphisms $\theta: B \rightarrow B^{\prime}, \eta: B^{\prime} \rightarrow A^{\prime}$ such that

(*) $\eta \theta=\alpha$

(**) $H_{B^{\prime} / A} \supset H_{C / A} \cdot B^{\prime}$.

Proof. Choose some elements $y^{\prime}=\left(y_{1}^{\prime}, \cdots, y_{N}^{\prime}\right)$ from $D$ lifting $\bar{\gamma}(y+$ $\left.d^{2 e+1} B\right)$. By iii) we get $\alpha(y)-\beta\left(y^{\prime}\right) \in d^{2 e+1} A^{\prime}$ and so there exists $y^{\prime \prime} \in d^{e} A^{N^{*}}$ such that

$$
\alpha(y)-\beta\left(y^{\prime}\right)=d^{e+1} y^{\prime \prime} .
$$

Since $d \in \Delta_{f}(y) \cap((f): \mathfrak{p})(y)$ by ii), there exist some $r \times r$-minors $\left\{M_{\jmath}\right\}_{j=1, \ldots, k}$ of the Jacobian matrix $J:=(\partial f / \partial y)$, some polynomials $\left\{L_{j}\right\}_{j=1, \ldots, k}$ from $A[Y]$ and a polynomial $P \in((f): \mathfrak{p})$ such that

$$
d=\sum_{j=1}^{k}\left(L_{j} M_{j}\right)(y)=P(y) .
$$

By iii) it follows

$$
\begin{aligned}
& d+\mathfrak{b}=\sum_{j=1}^{k}\left(L_{j} M_{j}\right)\left(\bar{\gamma}\left(y+d^{2 e+1} B\right)\right)=\sum_{j=1}^{k}\left(L_{j} M_{j}\right)\left(y^{\prime}\right)+\mathfrak{b}, \\
& d+\mathfrak{b}=P\left(\bar{\gamma}\left(y+d^{2 e+1} B\right)\right)=P\left(y^{\prime}\right)+\mathfrak{b}
\end{aligned}
$$

and so

$$
d-\sum_{j=1}^{k}\left(L_{j} M_{j}\right)\left(y^{\prime}\right) \in \mathfrak{b} \subset d^{2 e+1} C, \quad d-P\left(y^{\prime}\right) \in d^{2 e+1} C
$$

$\left(\right.$ see $\left.\left.\mathrm{iv}_{2}\right)\right)$. Then there exist two elements $s, s^{\prime} \in 1+d^{2 e} C$ such that

$$
s d=\sum_{j=1}^{k}\left(L_{j} M_{j}\right)\left(y^{\prime}\right), \quad s^{\prime} d=P\left(y^{\prime}\right)
$$

Like in (6.1) Step 1, we complete $J$ to a square matrix $H_{i}$ by adding some $(N-r)$-rows of the unit $N \times N$-matrix $I_{N}$ in order to get $\operatorname{det} H_{i}=M_{i}$ (if $r=N$ then $k=1$ and $H:=J$ ). Then there exists a $N \times N$-matrix $G_{i}^{\prime}$ over $A[Y]$ such that $H_{i} G_{i}^{\prime}=G_{i}^{\prime} H_{i}=M_{i} I_{N}$. Put $G_{i}:=L_{i} G_{i}^{\prime}$. We have 


$$
H_{i} G_{i}=G_{i} H_{i}=\left(M_{i} L_{i}\right) I_{N} .
$$

By (7.1.2) it results

$$
\sum_{i=1}^{k}\left(H_{i} G_{i}\right)\left(y^{\prime}\right)=\sum_{i=1}^{k}\left(G_{i} H_{i}\right)\left(y^{\prime}\right)=s d I_{N} .
$$

Denote $z^{(i)}:=H_{i}\left(\beta\left(y^{\prime}\right)\right) y^{\prime \prime} \in d^{e} A^{\prime v}$, We have $z_{j}^{(i)}=z_{j}^{(1)}$ for all $i=1, \cdots, k$ if $j \leq r$, because the first $r$-rows of $H_{i}$ form exactly the matrix $J$, From (7.1.4) it results

$$
\sum_{i=1}^{k} G_{i}\left(\beta\left(y^{\prime}\right)\right) z^{(i)}=\rho(s d) y^{\prime \prime}
$$

and so by (7.1.1)

$$
s \alpha(y)=s \beta\left(y^{\prime}\right)+d^{e} \sum_{i=1}^{k} G_{i}\left(\beta\left(y^{\prime}\right)\right) z^{(i)} .
$$

Let $Z=\left(Z_{1}^{(1)}, \cdots, Z_{r}^{(1)},\left\{Z_{j}^{(1)}\right\}_{\substack{1 \leq i \leq k \\ r<j \leq N}}\right), Z^{\prime}=\left(Z_{1}^{\prime}, \cdots, Z_{N}^{\prime}\right)$ be some variables and

$$
h_{j}:=s Y_{j}-s y_{j}^{\prime}-d^{e+1} Z_{j}^{\prime}-d^{e} \sum_{i=1}^{k} G_{i}\left(y^{\prime}\right) Z_{j}^{(i)},
$$

$j=1, \cdots, N$ some polynomials from $C\left[Y, Z, Z^{\prime}\right]$, where $Z_{j}^{(i)}:=Z_{j}^{(1)}$ when $j \leq r$. By (7.1.5) $\rho$ extends to an $A$-morphism $\eta^{\prime}: C\left[Y, Z, Z^{\prime}\right] /(p, h) \rightarrow A^{\prime}$, $h=\left(h_{1}, \cdots, h_{N}\right)$ given by $Y \backsim \alpha(y), Z \backsim z, Z^{\prime} \backsim 0$.

Let $m=\max \left\{\operatorname{deg} P, \max _{i=1, \ldots, r} \operatorname{deg} f_{i}\right\}$. Then $s^{m} f_{i}, s^{m} P$ can be expressed as some polynomials $\hat{f}_{i}, \hat{P}$ in $s Y$. Using (7.1.6) we get by Taylor's formula

$$
\begin{aligned}
& s^{m} P=\hat{P}(s Y) \equiv \hat{P}\left(s y^{\prime}\right) \bmod \left(d^{e}, h\right) \text { and } \\
& s^{m} f_{i}=\hat{f}_{i}(s Y) \equiv \hat{f}_{i}\left(s y^{\prime}\right)+\sum_{j=1}^{N} \frac{\partial \hat{f}}{\partial\left(s Y_{j}\right)}\left(s y^{\prime}\right)\left[d^{e+1} Z_{j}^{\prime}+d^{e} \sum_{v=1}^{k} G_{v}\left(y^{\prime}\right) Z_{j}^{(v)}\right] \\
&+d^{2 e} Q_{i}^{\prime} \bmod h, i=1, \cdots, r,
\end{aligned}
$$

where

$$
\frac{\partial \hat{f}}{\partial(s Y)}\left(s y^{\prime}\right)=s^{m-1} J\left(y^{\prime}\right) \quad \text { and } \quad Q^{\prime}=\left\{Q_{i}^{\prime}\right\}_{i=1, \ldots, r}
$$

is a system of polynomials from $C\left[Z, Z^{\prime}\right]$ containing only monomials of degree $\geq 2$. Thus

$$
\begin{aligned}
& s^{m} P \equiv s^{m} P\left(y^{\prime}\right) \bmod \left(d^{e}, h\right) \text { and } \\
& s^{m} f\left(y^{\prime}\right)+s^{m-1} d^{e} \sum_{v=1}^{k}\left(J G_{v}\right)\left(y^{\prime}\right) Z^{(v)}+d^{e+1} Q \equiv s^{m} f \bmod h,
\end{aligned}
$$


where $Q=s^{m-1} J\left(y^{\prime}\right) Z^{\prime}+d^{e-1} Q^{\prime}$. Note that $J G_{v}=\left(M_{v} L_{v}\right) E$, where $E:=$ $\left(I_{1} \mid 0\right)$; this follows from (7.1.3). It results

$$
\begin{array}{r}
\sum_{v=1}^{k}\left(J G_{v}\right)\left(y^{\prime}\right) Z^{(v)}=\sum_{v=1}^{k}\left(M_{v} L_{v}\right)\left(y^{\prime}\right) E Z^{(v)} \\
=\sum_{v=1}^{k}\left(M_{v} L_{v}\right)\left(y^{\prime}\right) E Z^{(1)}=s d E Z^{(1)}
\end{array}
$$

because $E Z^{(v)}=\left\{Z_{j}^{(v)}\right\}_{1 \leq j \leq r}=\left\{\boldsymbol{Z}_{j}^{(1)}\right\}_{1 \leq j \leq r}=E Z^{(1)}$.

Substituting (7.1.8) in (7.1.7) we get

$$
s^{m} f_{i}\left(y^{\prime}\right)+d^{e+1}\left[s^{m} Z_{i}^{(1)}+Q_{i}\right] \equiv s^{m} f_{i} \bmod h, i=1, \cdots, r .
$$

Since

$$
f\left(y^{\prime}+\mathfrak{b}\right)=f\left(\bar{\gamma}\left(y+d^{2 e+1} B\right)\right)=\bar{\gamma}\left(f(y)+d^{2 e+1} B\right)=0
$$

it follows $f\left(y^{\prime}\right) \in \mathfrak{b}$. By iv) there exist some elements $c=\left(c_{\imath}\right)_{i} \in C^{r}$ such that

$$
f_{i}\left(y^{\prime}\right)=d^{2 e+1} c_{\imath} \text {. }
$$

Denote $g_{i}:=s^{m} d^{e} c_{i}+s^{m} \boldsymbol{Z}_{i}^{(1)}+Q_{i} \in C\left[Z, Z^{\prime}\right]$. By (7.1.9) we get

$$
d^{e+1} g_{i} \equiv s^{m} f_{i} \bmod h, i=1, \cdots, r .
$$

Take $B^{\prime}:=C\left[Y, Z, Z^{\prime}\right] /(p, h, g), g=\left(g_{i}\right)_{i=1, \ldots, r}$ and let $\theta$ be the composite map $B \rightarrow C \otimes{ }_{A} B \cong C[Y] /(\mathfrak{p}) \rightarrow B^{\prime}$. Using (7.1.10) we get

$$
d^{e+1} \eta^{\prime}(g+(\mathfrak{p}, h))=\eta^{\prime}\left(d^{e+1} g+(\mathfrak{p}, h)\right)=\eta^{\prime}(0)=0
$$

and so

$$
\eta^{\prime}(g+(\mathfrak{p}, h)) \in \mathrm{Ann}_{A^{\prime}} d^{e+1} A^{\prime} .
$$

On the other hand it holds

$$
\rho\left(s^{m} d^{e} c\right)=g(0,0) \equiv g(z, 0)=\eta^{\prime}(g+(p, h)) \bmod z A^{\prime}
$$

Thus $\eta^{\prime}(g+(\eta, h)) \in d^{e} A^{\prime}$. By i) we deduce that

$$
d^{e} A^{\prime} \cap \mathrm{Ann}_{A^{\prime}} d^{e} A^{\prime}=(0)
$$

and so $\eta^{\prime}(g+(\eta, h))=0$. Thus $\eta^{\prime}$ induces an $A$-morphism $\eta: B^{\prime} \rightarrow A^{\prime}$.

Note that we have

$$
\left(\frac{\partial g_{i}}{\partial Z_{j}^{(1)}}\right)_{1 \leq i, j \leq r} \equiv s^{m} I_{r} \bmod d^{e-1}\left(Z, Z^{\prime}\right), \quad\left(\frac{\partial g}{\partial Y}\right)=0, \quad\left(\frac{\partial h}{\partial Y}\right)=s I_{s},
$$


where $I_{r}, I_{N}$ are the unit matrices of order $r$, respectively $N$. Consequently $\Delta_{(g, h)} B^{\prime}$ contains an element from $1+d B^{\prime}$. By (7.1.10) we get $s^{m} \in$ $\left(\left(d^{e+1} g, h\right):(f)\right)$ and so

$$
s^{m} P \in\left(\left(d^{e+1} g, h\right):(f)\right)((f): \mathfrak{p}) \subset\left(\left(d^{e+1} g, h\right): \mathfrak{p}\right) .
$$

We have

$$
s^{m} P \equiv s^{m} P\left(y^{\prime}\right)=s^{m} s^{\prime} d \equiv d \bmod \left(d^{e}, h\right)
$$

by (7.1.2) and (7.1.7). Thus there exists a polynomial $F \in C\left[Y, Z, Z^{\prime}\right]$ such that

$$
s^{m} P \equiv d\left(1+d^{e-1} F\right) \bmod h
$$

and so

$$
d\left(1+d^{e-1} F\right) \mathfrak{p} \subset\left(d^{e+1} g, h\right) .
$$

Denote $\tilde{B}:=C_{s}\left[Y, Z, Z^{\prime}\right] /(h)$. We have

$$
\left(1+d^{e-1} F\right) \hat{\beta} \tilde{B} \subset\left(d^{e} g\right) \tilde{B}+\operatorname{Ann}_{\tilde{B}} d
$$

On the other hand like in (7.1.7) it holds

$$
\mathfrak{p} \equiv \mathfrak{p}\left(y^{\prime}\right) \bmod \left(d^{e}, h\right) C_{s}\left[Y, Z, Z^{\prime}\right]
$$

and

$$
\mathfrak{p}\left(y^{\prime}+\mathfrak{b}\right)=\bar{\gamma}\left(\mathfrak{p}(y)+d^{2 e+1} B\right)=\bar{\gamma}(0)=0
$$

By $\mathrm{iv}_{2}$ ) it follows

$$
\mathfrak{p} \subset\left(h, d^{e}, \mathfrak{p}\left(y^{\prime}\right)\right) C_{s}\left[Y, Z, Z^{\prime}\right] \subset\left(h, d^{e}\right) C_{s}\left[Y, Z, Z^{\prime}\right]
$$

and so we get

$$
\left(1+d^{e-1} F\right) \mathfrak{p} \subset\left(d^{e} g\right) \tilde{B}+\operatorname{Ann}_{\tilde{B}} d \cap d^{e} \tilde{B} .
$$

If $x \in H_{C / A}$ then $C_{x}$ is a smooth $A$-algebra. Since $\tilde{B}$ is a smooth $C$-algebra we deduce that $\tilde{B}_{x}$ is a smooth $A$-algebra. By flatness we get from i)

$$
\operatorname{Ann}_{\tilde{B}_{x}} d^{e} \tilde{B}_{x}=\left(\operatorname{Ann}_{A} d^{e}\right) \tilde{B}_{x}=\left(\operatorname{Ann}_{A} d^{e+1}\right) \tilde{B}_{x}=\operatorname{Ann}_{\tilde{B}_{x}} d^{e+1} \tilde{B}_{x}
$$

and so

$$
d^{e} \tilde{B}_{x} \cap \operatorname{Ann}_{\tilde{B}_{x}} d^{e} \tilde{B}_{x}=(0) .
$$

From (7.1.12) we obtain 


$$
\left(1+d^{e-1} F\right) \mathfrak{p} \tilde{B}_{x} \subset\left(d^{e} g\right) \tilde{B}_{x} .
$$

Then there exists a positive integer $n$ such that

$$
(s x)^{n}\left(1+d^{e-1} F\right) \mathfrak{p} \subset\left(d^{e} g, h\right) \subset(g, h) C\left[Y, Z, Z^{\prime}\right]
$$

i.e. $\sqrt{((g, h): \mathfrak{p}) B^{\prime}}$ contains an element from $x+d B^{\prime}$. Thus $H_{B^{\prime} / C}$ contains an element from $x+d B^{\prime}$ and so

$$
H_{C / A} B^{\prime} \subset H_{B^{\prime} / C}+d B^{\prime} .
$$

Note that $\operatorname{det}\left(\partial h / \partial Z^{\prime}\right)=\left(-d^{e+1}\right)^{N}$ and so we have $d^{N(e+1)} \in \Delta_{h\left(y, z, z^{\prime}\right)}$ By (7.1.10) we get $d^{e+1} g \equiv 0 \bmod h C\left[Y, Z, Z^{\prime}\right] /(p)$ and so

$$
d^{e+1} \in\left(h\left(C \otimes_{A} B\right)\left[Z, Z^{\prime}\right]:(g)\right) .
$$

Thus $d \in H_{B^{\prime} / C \otimes_{A} B}$. By base change (Note (2.3)) one has $H_{C \otimes_{A} B / C} \supseteq$ $H_{B / A} \cdot C \otimes_{A} B$. Since $d \in H_{B / A}$ it follows

$$
d \in H_{B^{\prime} / C}
$$

(see Note (2.2)). From (7.1.14) we get

$$
H_{C / A} B^{\prime} \subset H_{B^{\prime} / C}
$$

and so $(* *)$ is now a consequence of Note (2.2).

Q.E.D.

\section{§8. The Lifting Lemma}

(8.1) Lifting Lemma. Let $A \rightarrow A^{\prime}$ be a ring morphism, $D:=A[Y]$ the polynomial A-algebra in $Y=\left(Y_{1}, \cdots, Y_{N}\right), d$ an element of $A, \mathfrak{b} \subset \mathfrak{a} \subset D$ two finitely generated ideals of $D$, e a positive integer, $\bar{D}:=D / \mathfrak{b}, \bar{A}:=A / d^{2 e} A$, $\bar{A}^{\prime}:=A^{\prime} / d^{2 e} A^{\prime}$ and $v: D \rightarrow A^{\prime}$ a morphism of $A$-algebras. Suppose that

i) $v(\mathfrak{b}) \subset d^{2 e} A$ and $d^{2 e} \in \mathfrak{b}$,

ii) $\mathfrak{a} / \mathfrak{b}=H_{\bar{D} / \bar{A}}$,

iii) $\mathrm{Ann}_{A} d^{e}=\mathrm{Ann}_{A} d^{e+1}, \mathrm{Ann}_{A^{\prime}} d^{e} A^{\prime}=\mathrm{Ann}_{A^{\prime}} d^{e+1} A^{\prime}$.

Then there exist a finite presentation A-algebra $C$ and an A-morphisri $w: C \rightarrow A^{\prime}$ such that

1) $C \supset D$ and $\left.w\right|_{D}=v$,

2) $\mathfrak{b} C \subset d^{e} C$,

3) $a \subset H_{C / A}$,

Proof. Let $y=\left(y_{1}, \cdots, y_{N}\right)$ be the residue class modulo $\mathfrak{b}$ of $Y$ in $D$ and $\hat{P}=\left(\hat{P}_{1}, \cdots, \hat{P}_{t}\right)$ a system of elements from a such that $\sqrt{(\hat{P})}=\mathfrak{a}$. 
By ii) we can choose $\hat{P}$ such that for each $j=1, \cdots, t$ there exists a system of polynomials $f_{j}=\left(f_{j 1}, \cdots, f_{j r_{j}}\right)$ from $\mathfrak{b}$, whose residue class $\bar{f}_{j}$ modulo $d^{2 e} D$ satisfies

$$
\left.\hat{P}_{j} \bar{D} \subset \Delta_{f_{j}}\left(\left(\bar{f}_{j}\right): \mathfrak{b} \bar{A}[Y]\right)\right)(y)
$$

Complete $\left(f_{j}\right)_{j}$ with some polynomials $f_{o}=\left(f_{o 1}, \cdots, f_{o r_{o}}\right)$ from $\mathfrak{b}$ in order to get a system of generators $f$ of $\mathfrak{b}$. Using i) there exist some elements $z=\left\{z_{j i} \mid 0 \leq j \leq t, 1 \leq i \leq r_{j}\right\}$ from $d^{e} A^{\prime}$ such that

$$
v\left(f_{j i}\right)=d^{e} z_{j i}, \quad 0 \leq j \leq t, \quad 1 \leq i \leq r_{j} .
$$

Denote $g_{j i}:=f_{j i}-d^{e} Z_{j i} \in D[Z], Z=\left(Z_{j i}\right), g_{j}=\left(g_{j i}\right)_{i}, g=\left(g_{j}\right)_{0 \leq j \leq t}$.

For every $j=1, \cdots, t$ there exist by (8.1.1) a polynomial $P_{j} \in \hat{P}_{j}+\mathfrak{b}$ such that

$$
P_{j} \in \Delta_{f_{j}} \cap\left(\left(f_{j}, d^{2 e}\right) D: \mathfrak{b}\right) .
$$

Let $\quad H=\left\{H_{\jmath u k i} \mid 0 \leq j, u \leq t, j \neq 0, j \neq u, 1 \leq i \leq r_{j}, 1 \leq k \leq r_{u}\right\}$,

$$
G=\left\{G_{j u k} \mid 0 \leq j, u \leq t, j \neq 0, j \neq u, 1 \leq k \leq r_{u}\right\}
$$

be some polynomials from $D$ such that

$$
\begin{aligned}
& P_{j} f_{u k}+\sum_{i=1}^{r_{j}} H_{j u k i} f_{j i}+d^{2 e} G_{j u k}=0, \\
& \quad 0 \leq j, u \leq t, j \neq 0, j \neq u, \quad 1 \leq k \leq r_{u}
\end{aligned}
$$

(this follows from (8.1.3)). Denote:

$$
F_{j u k}:=P_{j} Z_{u k}+\sum_{i=1}^{r j} H_{j u k i} Z_{j i}+d^{e} G_{j u k}, \quad j \neq 0, j \neq u .
$$

By (8.1.4) we get

$$
d^{e} F_{j u k}+P_{j} g_{u k}+\sum_{i=1}^{r_{j}} H_{j u k i} g_{j i}=0
$$

and so $F_{j u k}(v(Y), z) \in \operatorname{Ann}_{A^{\prime}} d^{e} A^{\prime}$. Since $F_{\jmath u k} \in\left(Z, d^{e}\right) D[Z]$ we get $F_{j u k}(v(Y), z)$ $\in d^{e} A^{\prime}$ and by iii)

$$
F_{\jmath u k}(v(Y), z) \in d^{e} A^{\prime} \cap \mathrm{Ann}_{A^{\prime}} d^{e} A^{\prime}=(0) .
$$

Take $C:=D[Z] /(g, F), F=\left(F_{\jmath u_{k}}\right)$ and let $w: C \rightarrow A^{\prime}$ be the $A$-morphism given by $Y \sim v(Y), Z \backsim z$. Clearly $d \in \sqrt{\Delta_{g}}$ and by (8.1.5) we get $d \in$ $((g):(F))$ and so $d \in H_{C / A}$. Since $P_{j} \in \Delta_{f_{j}}, 1 \leq j \leq t, P_{j} \in \sqrt{\Delta_{F_{j}}}$, 


$$
F_{j}=\left(F_{j u k}\right)_{\substack{1 \leq u \leq t, u \neq j \\ 1 \leq k \leq r u}} \text { and } \quad \frac{\partial g}{\partial Z} \equiv 0 \bmod d
$$

we get

$$
P_{j} \in \sqrt{\Delta_{\left(g_{j}, F_{j}\right)} C}+d C .
$$

By (8.1.5) we have

$$
\begin{aligned}
& -d^{e} P_{j} F_{\sigma \tau \lambda}=P_{\sigma}\left(P_{j} g_{\tau \lambda}\right)+\sum_{\mu=1}^{r_{\sigma}} H_{\sigma \tau \lambda \mu}\left(P_{j} g_{\sigma \mu}\right) \\
& -d^{e} F_{j \tau \lambda}=P_{j} g_{\tau \lambda}+\sum_{i=1}^{r j} H_{j \tau \lambda i} g_{j i} \equiv P_{j} g_{\tau \lambda} \bmod \left(g_{j}\right) \\
& -d^{e} F_{j \sigma \mu} \equiv P_{j} g_{\sigma \mu} \bmod g_{i} ; \quad 1 \leq \sigma, \tau \leq t, \sigma \neq \tau, 1 \leq \lambda \leq r_{\imath}, 1 \leq \mu \leq r_{\sigma}
\end{aligned}
$$

and so by substitution we get that

$$
R_{j \sigma \tau \lambda}=P_{j} F_{\sigma \tau \lambda}-P_{\sigma} F_{j \tau \lambda}-\sum_{\mu=1}^{r_{\sigma}} H_{\sigma \tau \lambda \mu} F_{j \sigma \mu} .
$$

satisfies

$$
d^{e} R_{\gamma \sigma \tau \lambda} \equiv 0 \bmod \left(g_{j}\right)
$$

Clearly $E_{\jmath}:=\left(D[Z] /\left(g_{j}\right)\right)_{P_{j}}$ is a smooth $A$-algebra and by flatness we get

$$
\mathrm{Ann}_{E_{j}} d^{e} E_{j}=\left(\mathrm{Ann}_{A} d^{e}\right) \cdot E_{j}=\left(\mathrm{Ann}_{A} d^{e+1}\right) E_{j}=\mathrm{Ann}_{E_{j}} d^{e+1} E_{\gamma} .
$$

Suppose for the moment that the following Lemma holds:

(8.2) Lemma. $P_{j} R_{j \sigma \tau \lambda} \equiv 0 \bmod \left(d^{e}, g_{j}\right) D[Z]$.

Then by (8.1.7) we have:

$$
P_{j} R_{j \sigma \tau} E_{j} \subset d^{e} E_{j} \cap \mathrm{Ann}_{E_{j}} d^{e} E_{j}=(0)
$$

and so there exists a positive integer $s \geq 1$ such that

$$
P_{j}^{s} R_{j \sigma \imath \lambda} \equiv 0 \bmod \left(g_{\jmath}\right) \text {. }
$$

Then one has

$$
P_{j}^{s+1} F_{\sigma \tau \lambda} \equiv P_{j}^{s} R_{j \sigma \tau \lambda} \equiv 0 \bmod \left(g_{j}, F_{j}\right) .
$$

for all $\sigma, \tau, \lambda$ and we obtain

$$
P_{\jmath} C \subset \sqrt{\left(\left(g_{j}, F_{j}\right):(F)\right) C} .
$$

As $P_{j} \in \sqrt{\left(\left(g_{j}, F_{j}\right):(g)\right)}$ by (8.1.5), it holds

$$
P_{j} C \subset \sqrt{\left(\left(g_{j}, F_{j}\right):(g, F)\right) C}
$$


and so $(P) \subset H_{C / A}$ (see (8.1.6)). Since $f \equiv d^{e} Z \bmod g$ it follows $\mathfrak{b} C=f C \subset d^{e} C$, i.e. 2). Then we have $\mathfrak{a}=\sqrt{(\hat{P})} \subset \sqrt{(P)+\mathfrak{b}} \subset \sqrt{ } H_{C / A}+d^{e} C=H_{C / A}$, i.e. 3).

Proof of Lemma (8.2). It is easy to note that

$$
S_{j \sigma \tau \lambda i}:=P_{\sigma} H_{j \tau \lambda \tau}+\sum_{\mu=1}^{r_{\sigma}} H_{\sigma: \lambda i l} H_{j \sigma \mu i}
$$

is a polynomial from $D$ satisfying

$$
R_{j \sigma \tau \lambda}+\sum_{i=1}^{r_{j}} S_{j \sigma \tau \lambda i} Z_{j i} \equiv 0 \bmod d^{e}
$$

Now by (8.1.4) we obtain

$$
\sum_{i=1}^{r_{j}} \sum_{\mu=1}^{r_{\sigma}} H_{\sigma \tau \lambda \mu} H_{j \sigma \mu i} f_{j i} \equiv-\sum_{\mu=1}^{r_{\sigma}} H_{\sigma \tau \lambda \mu}\left(P_{j} f_{\sigma \mu}\right) \equiv P_{j} P_{\sigma} f_{\tau \lambda} \equiv-P_{\sigma} \sum_{i=1}^{r_{j}} H_{j \tau \lambda i} f_{j i} \bmod d^{2 e}
$$

Then $\sum_{i=1}^{r j} S_{j \sigma \tau i i} f_{j i} \equiv 0 \bmod d^{2 e}$ and taking the derivations we get

$$
\sum_{i=1}^{r j} S_{j \sigma z \lambda i} \frac{\partial f_{j i}}{\partial Y} \equiv 0 \bmod \left(d^{2 e}, f_{j}\right) D
$$

It results

$$
\Delta_{f_{j}} S_{j \sigma \tau \lambda i} \subset\left(d^{2 e}, f_{j}\right) D \subset\left(d^{e}, g_{j}\right) D[Z] .
$$

In particular we obtain

$$
P_{\jmath} S_{\jmath \sigma \tau \lambda i} \in\left(d^{e}, g_{j}\right) D[Z]
$$

which is enough by (8.2.1).

Q.E.D.

\section{§9. Proof of Desingularization Lemma}

First we present the following

(9.1) Lemma. Let $A \rightarrow A^{\prime}$ be a ring morphism, $B$ a finite presentation A-algebra, $y=\left(y_{1}, \cdots, y_{N}\right)$ a system of generators of $B$ over $A, \mathfrak{p}$ the kernel of the map $A[Y] \rightarrow B, Y=\left(Y_{1}, \cdots, Y_{N}\right) \sim y, \alpha: B \rightarrow A^{\prime}$ an A-morphism, $d$ an element from $A, q \subset A^{\prime}$ a prime ideal containing $d A^{\prime}, e \geq 2$ a positive integer and $\bar{A}:=A / d^{4 e+2} A, \bar{B}:=\bar{A} \otimes_{A} B, \bar{A}^{\prime}:=\bar{A} \otimes_{A} A^{\prime}$. Suppose that:

i) $\mathrm{Ann}_{A} d^{e}=\mathrm{Ann}_{A} d^{e+1}, \mathrm{Ann}_{A^{\prime}} d^{e} A^{\prime}=\mathrm{Ann}_{A^{\prime}} d^{e+1} A^{\prime}$,

ii) there exists a system of polynomials $f=\left(f_{1}, \cdots, f_{r}\right)$ from $\mathfrak{p}$ such that $d \in \Delta_{f}((f): \mathfrak{p})(y)$,

iii) there exist a finite presentation $\bar{A}$-algebra $\bar{D}$ and two $\bar{A}$-morphisms $\bar{\beta}: \bar{D} \rightarrow \bar{A}^{\prime}, \bar{\gamma}: \bar{B} \rightarrow \bar{D}$ such that 
iii $\left._{1}\right) \alpha$ lifts $\bar{\beta} \bar{r}$,

iii $\left.{ }_{2}\right) \quad \alpha\left(H_{B / A}\right) \bar{A}^{\prime} \subset \sqrt{\bar{\beta}\left(H_{\bar{D} / \bar{A}}\right) \bar{A}^{\prime}} \not \subset q \bar{A}^{\prime}$.

Then there exists a finite presentation A-algebra $B^{\prime}$ and two A-morphisms $v: B \rightarrow B^{\prime}, w: B^{\prime} \rightarrow A^{\prime}$ such that

$$
\begin{aligned}
& (*) \quad w v=\alpha, \\
& (* *) \quad \alpha\left(H_{B / A}\right) \subset \sqrt{w\left(H_{B^{\prime} / A}\right) A^{\prime}} \not \subset q .
\end{aligned}
$$

Proof. Let $D$ be a polynomial $A$-algebra such that there exists a surjective $A$-morphism $\omega: D \rightarrow \bar{D}$ and take an $A$-morphism $\beta: D \rightarrow A^{\prime}$ lifting the composite map $D \stackrel{\omega}{\longrightarrow} \bar{D} \stackrel{\bar{\beta}}{\longrightarrow} \bar{A}^{\prime}$. Apply the Lifting Lemma to the case $A, A^{\prime}, D, d, \operatorname{Ker} \omega \subset \mathfrak{a}:=\omega^{-1}\left(H_{\bar{D} / \bar{A}}\right), e^{\prime}:=2 e+1, \beta$. Then there exist a finite presentation $A$-algebra $C$ and an $A$-morphism $\rho: C \rightarrow A^{\prime}$ such that

1) $C \supset D$ and $\left.\rho\right|_{D}=\beta$,

2) $\operatorname{Ker} \omega \subset d^{2 e+1} C$,

3) $\mathfrak{a} \subset H_{C / A}$,

Now, apply the Desingularization Principle to the case $A, A^{\prime}, B, \alpha, e$, $D, \operatorname{Ker} \omega, d, \beta, \bar{\gamma}, C, \rho$. Then there exist a finite type $C$-algebra $B^{\prime}$ and two $A$-morphisms $v: B \rightarrow B^{\prime}, w: B^{\prime} \rightarrow A^{\prime}$ such that

4) $w v=\alpha$,

5) $H_{B^{\prime} / A} \supset H_{C / A} B^{\prime}$.

Thus we have $w\left(H_{B^{\prime} / A}\right) \supset \beta(\mathfrak{a})$ bu 3) and $\beta(\mathfrak{a}) \not \subset q$ by $\left.\mathrm{iii}_{2}\right)$ : i.e., one holds $(* *)$.

Q.E.D.

(9.2) Conollary. Let $u: A \rightarrow A^{\prime}$ be a morphism of noetherian rings, $B$ a finite type A-algebra, $\alpha: B \rightarrow A^{\prime}$ and $A$-morphism, $q \subset A^{\prime}$ a prime ideal and $d \in u^{-1} q$ an element which is standard for $B$ over $A$. Then there exist a positive integer $n$ with the following property:

"Suppose that there exist a finite type $\bar{A}:=A / d^{n}$ A-algebra $\bar{D}$ and two $\bar{A}$-morphisms $\bar{\beta}: \bar{D} \rightarrow \bar{A}^{\prime}:=\bar{A} \otimes_{A} A^{\prime}, \bar{\gamma}: \bar{B} \rightarrow \bar{D}, \bar{B}:=\bar{A} \otimes_{A} B$ such that:

i) $\alpha$ lifts $\bar{\beta} \bar{\gamma}$

ii) $\alpha\left(H_{B / A}\right) \bar{A}^{\prime} \subset \sqrt{\bar{\beta}\left(H_{\bar{D} / \bar{A}}\right) \bar{A}^{\prime}} \not \subset q \bar{A}^{\prime}$.

Then there exist a finite type A-algebra $B^{\prime}$ and two A-morphisms $v: B \rightarrow B^{\prime}, w: B^{\prime} \rightarrow A^{\prime}$ which are subject to (*), (**) from Lemma (9.1)"'.

Proof. Since $d$ is a standard element for $B$ over $A$, there exists a system of generators $y=\left(y_{1}, \cdots, y_{N}\right)$ of $B$ over $A$ and a system of polynomials $f=\left(f_{1}, \cdots, f_{r}\right)$ from the kernel $I$ of the $A$-morphism $A[Y] \rightarrow B$, $Y=\left(Y_{1}, \cdots, Y_{N}\right) \leadsto y$ such that 


$$
d \in \sqrt{\left(\Delta_{f}((f): \Lambda)(y)\right.} .
$$

Thus we have

$$
d^{s} \in\left(\Delta_{f}((f): I)\right)(y)
$$

for a certain positive integer $s$. By noetherianity the ascending chains

$$
\begin{aligned}
& \operatorname{Ann}_{A} d \subset \cdots \subset \operatorname{Ann}_{A} d^{t} \subset \ldots \\
& \operatorname{Ann}_{A^{\prime}} u(d) \subset \cdots \subset \operatorname{Ann}_{A^{\prime}} u\left(d^{t}\right) \subset \cdots
\end{aligned}
$$

stop and so there exists a positive integer $e$ such that

$$
\mathrm{Ann}_{A} d^{e}=\mathrm{Ann}_{A} d^{e+1}, \quad \mathrm{Ann}_{A^{\prime}} u\left(d^{e}\right)=\mathrm{Ann}_{A^{\prime}} u\left(d^{e+1}\right) .
$$

Take $n:=4 e s+2 s$. By the above Lemma applied for $d^{s}$, the integer $n$ has the wanted property.

(9.3) Lemma. Let $u: A \rightarrow A^{\prime}$ be a morphism of noetherian rings, $B$ a finite type A-algebra, $\alpha: B \rightarrow A^{\prime}$ an A-morphism, $a \subset A^{\prime}$ an ideal contained in $\sqrt{\alpha\left(H_{B / A}\right) A^{\prime}}$ and $q$ a minimal prime ideal of $A^{\prime}$. Suppose that

i) all minimal prime over-ideals of a are minimal in $A^{\prime}$,

ii) $q \supset a$,

iii) there exist a finite type A-algebra $B_{1}$ and two $A$-morphisms $v_{1}: B$ $\rightarrow B_{1}, w_{1}: B_{1} \rightarrow A^{\prime}$ such that

iii $\left.{ }_{1}\right) \quad w_{1} v_{1}=\alpha$,

$\left.\mathrm{iii}_{2}\right) \quad w_{1}\left(H_{B_{1} / A}\right) \not \subset q$.

Then there exist a finite type A-algebra $B^{\prime}$ and two $A$-morphisms $v_{1}: B \rightarrow B^{\prime}$, $w: B^{\prime} \rightarrow A^{\prime}$ such that

$$
(+) \quad w v=\alpha \quad \text { and } \quad a \subset \sqrt{w\left(H_{B^{\prime} / A}\right) A^{\prime}} \not \subset q .
$$

Proof. Let $B_{1} \cong B[X] /(f), X=\left(X_{1}, \cdots, X_{n}\right), f=\left(f_{1}, \cdots, f_{m}\right)$ be a presentation of $B_{1}$ over $B$, the isomorphism being given by $X \rightarrow x \in B_{1}^{n}$. Let $p_{1}=q, \cdots, p_{e}$ be the minimal prime ideals from $A^{\prime}$ and $S:=A^{\prime} \backslash \bigcup_{i=1}^{e} p_{i}$. Suppose that $\left\{p_{i}\right\}_{1 \leq i \leq r}, r \leq e$ are exactly the minimal prime ideals associated to a. As $S^{-1} A^{\prime} \cong \prod_{i=1}^{e} A_{p_{i}}^{\prime}$ there exists an element $\omega=y^{\prime} / t^{\prime} \in S^{-1} A^{\prime}, y^{\prime} \in A^{\prime}$, $t^{\prime} \in S$ corresponding to $(\underbrace{1, \cdots, 1}_{p}, 0 \cdots 0)$ by previous isomorphism. Then $\omega^{2}=\omega$ and one has $t^{\prime \prime 2}\left(y^{\prime 2}-t^{\prime} y^{\prime}\right)=0$ for a certain $t^{\prime \prime} \in S$. Clearly $y:=t^{\prime \prime} y^{\prime}$, $t:=t^{\prime} t^{\prime \prime}$ hold in $A^{\prime}$
1) $y^{2}=t y$,
2) $y \notin p_{i}$ for $i, 1 \leq i \leq r$, 
3) the morphisms $A^{\prime} \rightarrow A_{p_{i}}^{\prime}, r<i \leq e$ map $y$ in 0 .

Take $D:=A[Y, T] /\left(Y^{2}-T Y\right), C:=D \otimes{ }_{A} B$ and $B^{\prime}:=C[X] /(Y f)$. Let $\gamma: D$ $\rightarrow A^{\prime}$ be the $A$-morphism given by $Y \rightarrow y, T \rightarrow t ; \beta: C \rightarrow A^{\prime}, \tilde{w}: C \otimes_{B} B_{1} \rightarrow A^{\prime}$ the maps given by $\gamma, \alpha$ resp. $\beta, w_{1}$ and $v, w$ the composite maps $B \rightarrow C$ $\rightarrow B^{\prime}$ resp. $B^{\prime} \rightarrow C[X] /(f)=C \otimes_{B} B_{1} \stackrel{\tilde{w}}{\longrightarrow} A^{\prime}$. Clearly $w v=\alpha$ and $B_{w^{-1} q}^{\prime} \cong$ $\left(C \otimes_{B} B_{1}\right)_{\tilde{w}-1 q}$ because $y \notin q$ by 2$)$. As $C \otimes_{B} B_{1} \cong D \otimes_{A} B_{1}$ we get $B_{w-1 q}^{\prime}$ smooth over $D$ (see $\left.\mathrm{iii}_{2}\right)$ ). But $D_{Y}$ is smooth over $A$ and so $B_{w-1 q}^{\prime}$ is smooth over $A$ too.

If $r=e$ we are ready. Otherwise we must show that $B_{w-1}^{\prime} p_{i}, r \leq i \leq e$ are smooth $A$-algebras. Fix an $i, r<i \leq e$. As $Y / T$ induces an idempotent in $B_{T}^{\prime}$ we get

$$
B_{T}^{\prime} \cong B_{T}^{\prime} /(T-Y) B_{T}^{\prime} \times B_{T}^{\prime} / Y B_{T}^{\prime} .
$$

Since $t, t-y \notin p_{i}$ we get $B_{w-1 p_{i}}^{\prime} \cong\left(B^{\prime} / Y B^{\prime}\right)_{w-1 p_{i}}$. But $B^{\prime} / Y B^{\prime} \cong B[X, T]$ is a smooth $B$-algebra and so $B_{w^{-1} p_{i}}^{\prime}$ is a smooth $B$-algebra too. Now, by our assumptions $B_{\alpha-1 p_{i}}$ is smooth over $A$. Thus $B_{w-1 p_{i}}^{\prime}$ is smooth over $A$

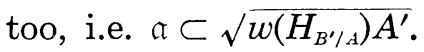

Q.E.D.

(9.4) Corollary. Let $u: A \rightarrow A^{\prime}$ be a morphism of noetherian rings, $B$ a finite type A-algebra, $\alpha: B \rightarrow A^{\prime}$ an A-morphism $\mathfrak{a} \subset A^{\prime}$ an ideal contained in $\sqrt{\alpha\left(H_{B / A}\right) A^{\prime}}$ and $q \supset \mathfrak{a}$ a minimal prime ideal of $A^{\prime}$. Suppose that

i) all minimal prime over-ideals of a are minimal in $A^{\prime}$,

ii) the field extension $k\left(u^{-1} q\right) \subset k(q)$ is separable and $\left(u^{-1} q\right) A_{q}^{\prime}=q A_{q}^{\prime}$.

iii) the map $A \rightarrow A_{q}^{\prime}$ given by $u$ is flat.

Then there exist a finite type $A$-algebra $B^{\prime}$ and two $A$-morphisms $v: B \rightarrow B^{\prime}$ $w: B^{\prime} \rightarrow A^{\prime}$ which are subject to (+) from Lemma (9.3).

For proof apply the above Lemma; its condition iii) holds by Corollary (3.3) (see our conditions ii), iii)).

(9.5) Lemma. Let $u: A \rightarrow A^{\prime}$ be a morphism of noetherian rings, $B$ a finite type A-algebra, $\alpha: B \rightarrow A^{\prime}$ a morphism of A-algebras, $\mathfrak{a} \subset A^{\prime}$ an ideal contained in $\sqrt{\alpha\left(H_{B / A}\right) A^{\prime}}$ and $q$ a minimal prime over-ideal of a. Suppose that:

i) all minimal prime over-ideals of a are still minimal prime overideals of $\left(u^{-1} \mathfrak{a}\right) A^{\prime}$,

ii) for every minimal prime over-ideal $p$ of a the map $A \rightarrow A_{p}^{\prime}$ is flat,

iii) it holds either 
$\left.\mathrm{iii}_{1}\right)$ the field extension $k\left(u^{-1} q\right) \subset k(q)$ is separable and $\left(u^{-1} q\right) A_{q}^{\prime}=q A_{q}^{\prime}$, or

iii $\left.{ }_{2}\right)$ for every positive integer $r$ there exist a finite type $\bar{A}_{r}:=A /\left(u^{-1} q\right)^{r}$ algebra $\bar{D}_{r}$ and two $\bar{A}_{r}$-morphisms $\bar{v}_{r}: \bar{B}_{r} \rightarrow \bar{D}_{r}, \bar{B}_{r}:=\bar{A}_{r} \otimes_{A} B, \bar{w}_{r}: \bar{D}_{r} \rightarrow$ $\bar{A}_{r}^{\prime}:=\bar{A}_{r} \otimes_{A} A^{\prime}$ such that $\bar{w}_{r}\left(H_{\bar{r}_{r} / \bar{A}_{r}}\right) \not \subset q \bar{A}_{r}^{\prime}$ and $\bar{w}_{r} \bar{v}_{r}=\bar{B}_{r} \otimes_{B} \alpha$.

Then there exist a finite type A-algebra $B^{\prime}$ and two A-morphisms $v: B \rightarrow B^{\prime}, w: B^{\prime} \rightarrow A^{\prime}$ which are subject to $(+)$ from Lemma (9.3).

Proof. Let $t_{\mathrm{a}} ;=\max \left\{\operatorname{ht} p \mid p \in \operatorname{Min}\left(A / u^{-1} \mathfrak{a}\right)\right\}$. Apply induction on $t_{\mathrm{a}}$. If $t_{a}=0$ then we claim that all minimal prime over-ideals of $\mathfrak{a}$ are minimal in $A^{\prime}$. Indeed, a minimal prime over-ideal $p$ of $\mathfrak{a}$ is still a minimal prime over-ideal of $\left(u^{-1} \mathfrak{a}\right) A^{\prime}$ (see i)). By [M] (13. B) (see ii)) we get

$$
\operatorname{ht}(p)=\operatorname{ht}\left(u^{-1} p\right),
$$

$p$ being also a minimal prime over-ideal of $\left(u^{-1} p\right) A^{\prime}$. If $p^{\prime} \supset u^{-1} \mathfrak{a}$ is a prime ideal contained in $u^{-1} p$ then $p$ is a minimal prime over-ideal of $p^{\prime} A^{\prime}$ by i) and applying Bourbaki Theorem [M] (9. B) for the flat map $A \rightarrow A_{p}^{\prime}$ we get $u^{-1} p=p^{\prime}$. Thus $u^{-1} p$ is a minimal prime over-ideal of $u^{-1} a$ and so

$$
\operatorname{ht}\left(u^{-1} p\right)=0
$$

$\left(t_{\mathrm{a}}=0\right)$ which proves our claim. Then this case is a consequence of Corollary (9.4) when $\mathrm{iii}_{1}$ ) holds, or Lemma (9.3) when $\mathrm{iii}_{2}$ ) holds for a suitable $r$ such that $\left(u^{-1} q\right)^{r} A_{u^{-1} q}=(0)$.

Now suppose $t_{a}>0$. Since $u^{-1} \mathfrak{a}$ is not contained in the union of $\left\{p^{\prime} \in \operatorname{Min} A \mid p^{\prime} \ngtr u^{-1}(\mathfrak{a})\right\}$ we can choose an element $d \in u^{-1}(\mathfrak{a})$ which is not contained in any minimal prime ideals of $A$ except in those containing $u^{-1} \mathfrak{a}$ (if there exist any). Clearly $t_{a^{\prime} / d^{s^{\prime}}}<t_{a}$ for every positive integer $s$.

Applying Lemma (2.4) there exist an $A$-algebra $B_{1}$ and two $A$-morphisms $v_{1}: B \rightarrow B_{1}, \alpha_{1}: B_{1} \rightarrow A^{\prime}$ such that

1) $\alpha_{1} v_{1}=\alpha$,

2) $H_{B_{1 / B}} \supset v_{1}\left(H_{B / A}\right)$.

3) $d \in H_{B_{1} / A}$.

Changing $(B, \alpha)$ by $\left(B_{1}, \alpha_{1}\right)$ we reduce our problem to the case when $d \in H_{B / d}$. Moreover using Lemma (3.4) we can reduce similarly to the case when $d$ is a standard element for $B$ over $A$. Let $n$ be the positive integer associated to $B, \alpha, d$ by Corollary (9.2). By induction hypothesis there exist an $\bar{A}:=A / d^{n} A$-algebra $\bar{D}$ and two $\bar{A}$-morphisms $\bar{\beta}: \bar{D} \rightarrow \bar{A}^{\prime}:=\bar{A} \otimes_{A} A^{\prime}$, 
$\bar{\gamma}: \bar{B} \rightarrow \bar{D}, \bar{B}:=\bar{A} \otimes_{A} B$ satisfying i), ii) from Corollary (9.2) [the induction hypothesis work on $\overline{\mathfrak{a}}:=\mathfrak{a} / d^{n} A^{\prime} \subset \sqrt{\bar{\alpha}\left(H_{B / A}\right) A^{\prime}}$ (see Note (2.3)) because $t_{\bar{a}}<t_{a}, \bar{\alpha}$ being given by $\alpha$ ]. This finishes our proof.

Q.E.D.

(9.5.1) Note. Actually in this paper we use Lemma (9.5) only when iii $_{1}$ ) holds, but when we work without conditions of separability we can have only $\mathrm{iii}_{2}$ ) fulfilled.

The possibility to apply Lemma (9.5) in the proof of Desingularization Lemma is given by the following

(9.6) Lemma. Let $A$ be a noetherian semilocal ring, $\left(q_{i}\right)_{1 \leq i \leq e}$ its maximal ideals, $u: R \rightarrow A$ a flat ring morphism, $s_{i}:=\operatorname{ht}\left(q_{i}\right)-\operatorname{ht}\left(u^{-1} q_{i}\right)$, $s=\sum_{1 \leq i \leq e} s_{i}$ and $\left(z_{i j}\right)_{\substack{1 \leq i \leq e \\ 1 \leq j \leq s-s_{i}}}$ some elements from $A$. Suppose that for every $i, 1 \leq i \leq e$

i) the local rings of $\left(q_{i}\right)_{i}$ on the fiber, i.e. $\left(A /\left(u^{-1} q_{i}\right) A\right)_{q_{i}}$, are regular,

ii) $z_{i}=\left(z_{i j}\right)_{1 \leq j \leq s-s i}$ induces in $A / q_{i}$ a system $\bar{z}_{i}$ of elements algebraically independent over $R / u^{-1} q_{i}$.

Then there exist a polynomial $R$-algebra $D$ and a flat R-morphism $v$ : $D \rightarrow A$ such that

1) the ideal $a:=\operatorname{Rad} A$ satisfies $\left(v^{-1} a\right) A_{q_{i}}=q_{i} A_{q_{i}}$ for all $i, 1 \leq i \leq e$,

2) $k\left(v^{-1} q_{i}\right)=k\left(u^{-1} q_{i}\right)\left(\bar{z}_{i}\right)$ for every $i, 1 \leq i \leq e$.

Proof. Apply induction on $s$. If $s=0$ then $D:=R, v:=u$ work. Suppose that $s>0$ and let $i, 1 \leq i \leq e$ be such that $s_{\imath}>0$. Then

$$
q_{i} \not \subset\left(u^{-1} q_{i}\right) A_{q_{i}}+q_{i}^{2} A_{q_{i}}
$$

by Nakayama's Lemma and we can choose an element $y_{i} \in q_{i}$ which is not contained in $\left(u^{-1} q_{i}\right) A q_{i}+q_{i}^{2} A_{q_{i}}$. For $j \neq i, 1 \leq j \leq e$ take $y_{j}:=z_{j 1}$.

By Chinese Remainder Theorem the canonical map

$$
f: A \longrightarrow \sum_{i=1}^{e} A / q_{i}^{2}+\left(u^{-1} q_{i}\right) A
$$

is surjective and so there exists an element $x \in A$ such that $f(x)$ is induced by $y=\left(y_{1}, \cdots, y_{e}\right)$.

Take $S:=R[X]$ and let $g: S \rightarrow A$ be the $R$-moxphism given by $X \backsim x$. By construction $\left(A / x A+u^{-1} q_{i}\right)_{q_{i}}$ is a regular ring of dimension $s_{i}-1$. As $g^{-1}\left(q_{j}\right)=u^{-1}\left(q_{j}\right) S$ for $j \neq i, i \leq j \leq e$, the Dimension Formula holds for $S \stackrel{q}{\longrightarrow} A$ and by [M] (21. D) we get flat the maps $\left.\left(S / u^{-1} q_{\lambda}\right) S\right)_{g-1 q_{\lambda}} \rightarrow A_{q_{\lambda}}$ for all $\lambda, 1 \leq \lambda \leq e$. Using [M] (20.G) we obtain the flatness of the maps 
$S_{g^{-1 q_{\lambda}}} \rightarrow A_{q_{\lambda}}$ induced by $g, 1 \leq \lambda \leq e$. Thus $g$ is flat. By induction hypothesis the result is stated for $S, g, A$ and so there exist $(D, v)$.

Q.E.D.

(9.7) Proof of Desingularization Lemma (5.1). Let $\left\{p_{i}\right\}_{1 \leq i \leq e}, p_{i} \subset A^{\prime}$, $q=p_{e}$ be the minimal prime over-ideals of a. Denote $s_{i}:=\operatorname{ht}\left(p_{i}\right)-$ ht $\left(u^{-1} p_{i}\right), 1 \leq i \leq e, s=\sum_{i=1}^{e} s_{i}, A^{\prime \prime}:=A^{\prime}[X], X=\left(X_{1}, \cdots, X_{s}\right), p_{i}^{\prime \prime}:=p_{i} A^{\prime \prime}$ and let $h: A^{\prime} \rightarrow A^{\prime \prime}$ be the inclusion. By Lemma (9.6) applied to $A, T^{-1} A^{\prime \prime}$, $T:=A^{\prime \prime} \backslash \bigcup_{i=1}^{e} p_{i}^{\prime \prime}, z_{i}:=\left(X_{1}, \cdots, X_{s-s_{i}}\right)$ there exist a polynomial $A$-algebra $R$ and an $A$-morphism $g: R \rightarrow A^{\prime \prime}$ such that for every $i, 1 \leq i \leq e$

$A$-algebra $R$ and an $A$-morphism $g: R \rightarrow A^{\prime \prime}$ such that for every $i, 1 \leq i \leq e$

1) $g^{-1}(\mathfrak{a}) A_{p_{i}^{\prime \prime}}^{\prime \prime}=p_{\imath} A_{p_{i}}^{\prime \prime \prime}$

2) the map $R \rightarrow A_{p_{i}^{\prime \prime}}^{\prime \prime}$ given by $g$ is flat,

3) $k\left(g^{-1}\left(p_{i}^{\prime \prime}\right)\right)=k\left(u^{-1}\left(p_{i}\right)\right)\left(X_{1}, \cdots, X_{s-s_{i}}\right)$ and using our hypotheses the extension $k\left(g^{-1}\left(p_{e}^{\prime \prime}\right)\right) \subset k\left(p_{e}^{\prime \prime}\right)$ is separable.

Using 1) note that $\left\{p_{i}^{\prime \prime}\right\}$ are minimal prime over-ideals of $\left(g^{-1} \mathfrak{a}\right) A^{\prime \prime}$.

Denote $B^{\prime \prime}:=R \otimes_{A} B$ and let $f^{\prime \prime}: B^{\prime \prime} \rightarrow A^{\prime \prime}$ be the $R$-morphism given by $g$ and $h f$. Apply Lemma (9.5) to the case $\left(R, g, A^{\prime \prime}, B^{\prime \prime}, f^{\prime \prime}, q A^{\prime \prime}\right)$. Thus there exist a finite type $A$-algebra $B^{\prime}$ and two $A$-morphisms $v: B^{\prime \prime} \rightarrow B^{\prime}$, $w^{\prime}: B^{\prime} \rightarrow A^{\prime \prime}$ such that

4) $w^{\prime} v=h f^{\prime \prime}$,

5) $\mathfrak{a} \subset \sqrt{w^{\prime}\left(H_{B^{\prime} A}\right) A^{\prime \prime}} \not \subset q A^{\prime \prime}$,

because $H_{B^{\prime \prime} / R} \supset H_{B / A} B^{\prime \prime}$. Since $A^{\prime} / q$ is not finite there exists some elements $x=\left(x_{1}, \cdots, x_{s}\right) \in A^{\prime s}$ such that the $A^{\prime}$-morphism $\sigma_{x}: A^{\prime \prime} \rightarrow A^{\prime}, X \rightarrow x$ satisfies

$$
\sigma_{x} w^{\prime}\left(H_{B^{\prime} / A}\right) \not \subset q
$$

and we can take $w:=\sigma_{x} w^{\prime}$. This follows from the following elementary

(9.7.1) Lemma. Let $A[X]$ be the polynomial ring in some variables $X=\left(X_{1}, \cdots, X_{n}\right)$ over a noetherian ring $A, q \subset A$ a prime ideal such that $A / q$ is not finite and $h \in A[X]$ a polynomial which is not in $q A[X]$. Then there exists a system of elements $x=\left(x_{1}, \cdots, x_{n}\right)$ from $A$ such that the $A$ morphism $\sigma_{x}: A[X] \rightarrow A$ satisfies

$$
\sigma_{x}(h) \notin q .
$$

For the proof of Lemma (9.7.1) apply induction on $n$; when $n=1$ note only that the residue $\bar{h}$ of $h$ modulo $q A[X]$ has just a finite number of solutions in $A / q$.

Q.E.D. 
Added in proof. The separable condition i) is not necessarily in Theorem (5.2) as shows our paper "General Néron desingularization and approximation" (to appear in Nagoya Math. J. Vol. 104 (1986)) which contains also some applications. Based on this result the Conjecture (1.5) was settled by M. Cipu and the author in Ann. Univ. Ferrara, ser. VII. Vol XXX (1984), 63-76.

\title{
REFERENCES
}

[A] Artin, M., Algebraic structure of power series rings, Contemp. Math. Soc., Providence (1982), 223-227.

[AD] Artin, M. and Denef, J., Smoothing of a ring homomorphism along a section, Arithmetic and Geometry, vol. II, Birkhäuser, Boston (1983), 5-32.

[CP] Cipu, M. and Popescu, D., Some extensions of Néron's p-desingularization and approximation, Rev. Roumaine Math. Pures Appl., t. XXIV, 10 (1981), 1299-1304.

[E] Elkik, R., Solutions d'equations à coefficients dans un anneaux hensélien, Ann. Sci. Ecole Norm Sup. $4^{\mathrm{e}}$ serie, t. 6 (1973), 533-604.

[KMPPR] Kurke, H., Mostowski, T., Pfister, G., Popescu, D. and Roczen, M., Die Approximationseigenschaft lokaler Ringe, Lect. Notes in Math., 634, SpringerVerlag, Berlin, 1978.

[M] Matsumura, H., Commutative algebra, Benjamin, New York, 1980.

[N] Néron, A., Modèles minimaux des variétés abéliennes sur les corps locaux et globaux, Publ. Math. IHES, 21, 1964.

[Ni] Nica, V., A desingularization theorem in non normal one dimensional rings, Preprint INCREST nr. 43/1983.

[Pl] Ploski, A., Note on a theorem of M. Artin, Bull. Acad. Polon. des Sci., t. XXII, 11 (1974), 1107-1110.

[P $\left.\mathrm{P}_{1}\right] \quad$ Popescu, D., A remark on two dimensional local rings with the property of approximation, Math. Z., 173 (1980), 235-240.

$\left[\mathrm{P}_{2}\right] \quad-$, Global form of Néron's $p$-desingularization, Proceedings "Weak of Algebraic Geometry", Bucharest, June 30-July 6, 1980, Teubner Texte, Band 40, Leipzig, 1981.

$\left[\mathrm{P}_{3}\right] \quad-$, Higher dimensional Néron desingularization and approximation Preprint INCREST nr. 50/1982.

$\left[\mathrm{P}_{4}\right] \quad-$ On Zariski's Uniformization Theorem, Algebraic Geometry, Bucharest 1982, Proceedings, Lect. Notes in Math., 1056, Springer-Verlag, Berlin, 1984.

[Z] Zariski, O., Local uniformization on algebraic varieties, Ann. of Math., 41 (1940).

\author{
INCREST \\ Department of Mathematics \\ Bd. Păcii 220, 79622 \\ Bucharest, Romania \\ and \\ Institute of Mathematics \\ Str. Academiei 14 \\ 70109 Bucharest, Romania
}

